\title{
L'exploration en imagerie par résonance magnétique des articulations temporo-mandibulaires
}

\author{
Nuclear magnetic resonance imaging exploration of the temporo-mandibular-joint
}

\author{
HÉDI ROMDANE*, CARLOS MADRID**, GILBERT DE MELLO*, BRUNO COURTOIS**
}

\begin{abstract}
RÉSUMÉ
L'articulation temporo-mandibulaire de nature diarthrosique, est située entre deux entités anatomiques l'une fixe le crâne et l'autre mobile la mandibule séparées par un disque. Cette articulation est mue par un complexe musculo-ligamentaire environnant. Cet ensemble est sollicité en permanence. Sa symptomatologie en cas de pathologie est multiple et variée. Elle est le reflet de nombreuses affections. L'étude réalisée s'est attachée, grâce à une revue de la littérature, à évoquer d'une façon exhaustive les différentes situations cliniques en rapport avec cette articulation et à chercher à définir la place de l'Imagerie par Résonance Magnétique (IRM) dans son exploration non sans rappeler les avantages et les inconvénients des méthodes conventionnelles telles que la tomodensitométrie, l'endoscopie, la scintigraphie, l'arthrotomographie et la chirurgie. L'IRM même si elle se présente comme la méthode non invasive par excellence n'est pas une panacée. Son indication doit se faire à bon escient. C'est ainsi qu'elle prend toute sa dimension grâce à un choix judicieux des séquences, dans l'examen paraclinique des dysfonctionnements crânio-mandibulaires où elle n'intervient que dans la confirmation du diagnostic, le bilan de gravité, le bilan pré ou post chirurgical, ou le contrôle de l'efficacité du traitement chirurgical car elle permet de visualiser tous les éléments anatomiques environnants grâce à l'émission de signaux dont l'intensité varie selon le type de tissu. Dans bon nombre de pathologies telles que les maladies rhumatoïdes ou congénitales l'IRM s'avère discutable en l'absence d'une véritable spécificité des images. L'indication de cette méthode d'exploration doit prendre en compte également de certains aspects collatéraux mais non moins importants tels que : le rendement attendu en fonction de la pathologie suspectée cliniquement, l'agressivité des examens de substitutions, le rapport coût/bénéfice comparé à celui des autres investigations possibles (Med Buccale Chir Buccale 2002;8;7-36).
\end{abstract}

mots clés : IRM, ATM, Diagnostic, Pathologies

médecine buccale chirurgie buccale VOL. $8, \mathrm{~N}^{\circ}$ 2002

\section{SUMMARY}

The Temporo-Mandibular J oint (TMJ ) diarthrosis by nature, is situated between two anatomical entities, one fixe the cranium, the other mobile, the mandibule. The articular surfaces of these two components are in congruence by means of the disc which role in the mandibular movement is major, for it lets the condyle free in its movements like sliding, turning, and revolving under loading. TMJ, permanently sollicited, is moved by musculo-ligamental complex. Its symptomatology in pathologic cases is numerous, it is the reflect of several affections which the origin could be inflammatory, traumatic, mecanic, functionnal or abnormalitive. This study is attached, thanks to a review of the literature, to evocate in an exhaustive way, the different clinical situations linked with this joint and to search how to define the position of MRI in its exploration.This, without reminding the advantages and drawbacks of the conventionnal procedures as CTscanning, endoscopy, scintigraphy, arthrotomography, and surgery. MRI, even whether it is a non-invasive procedure above all else, however, it is not a cure-all. It has to be practiced wisely. Actually this procedure of exploration shows his value, with a judicious choice of sequences in paraclinical test of cranio-mandibular disorders. It is useful in the confirmation of diagnosis, the gravity of the outcome, the pre and postsurgical outcome, or the controle of efficiency of surgical treatment, for it is able to visualize the main anatomical components linked with these disorders particulary the disc and its movement. In this aim we are attached ourselves to draw up a draft test easy, precise, and reprodicible in in both of static and dynamic methods. However, it is useful to support that for others pathologies as rhumatoid or congenital deseases, MRI seemsdebatable without any real specificity images. Finally, in a practical point of view it is major that the odontologist strives to considerate some important elements wich adjo in the indications of this explorative procedure. It concerns to take in consideration: the waited output in relation with the pathology clinically suspected, the violence of substitution tests, the ratio cost/profit compared to the one of other possible investigations (Med Buccale Chir Buccale 2002;8;7-36).

key words: MRI, TMJ , Diagnosis, Disorders

\footnotetext{
* Service de chirurgie buccale, Centre Hospitalier Régional Universitaire, Rennes, France.

** Département des Sciences anatomiques, UFR Odontologie, Toulouse, France.
}

Demande de tirés à part :

Hédi Romdane 9, rue du Vieux Saint Louis BP $1513 \quad 53015$ Laval cedex 15

Article reçu le 29 septembre 1999. Accepté pour publication le 13 mars 2001. 
médecine buccale chirurgie buccale

L. $8, \mathrm{~N}^{\circ} 1$

2002

page 8
Les troubles de l'articulation temporo-mandibulaire (ATM) sont un sujet croissant de consultation en odontologie. Longtemps, seul le compartiment dentaire, celui qui est à la vue, a été l'objet de l'attention diagnostique et thérapeutique. Puis, le refus de considérer la partie crâniale de l'articulation en aveugle ou par défaut a suscité des investigations d'une agressivité croissante : incidences radiologiques conventionnelles, tomographies, arthrographies et même arthroscopies interventionnelles. L'ère de la tomodensitométrie (TDM) maxillo-faciale a offert des images spectaculaires des structures osseuses de l'ATM mais avec la rançon d'une irradiation qui, si elle ne soulève que peu d'inquiétude en France, fait l'objet de suspicion dans les pays de culture scandinave ou anglosaxonne. Par ailleurs cette imagerie TDM ne fournit, malgré des essais méritoires, aucune information fiable sur une structure dont l'implication est toujours mieux reconnue dans la pathologie de l'ATM, le disque articulaire.

La résonance magnétique nucléaire est une technique d'analyse physico-chimique ancienne développée dès 1946 par Bloch [1] et Purcell [2]. L'application au champ médical est ainsi inaugurée en 1971 par Damadian R. [3]. Helms et coll [4], furent les premiers auteurs à avoir publié en 1984 l'application de l'imagerie par résonance magnétique (IRM) à l'exploration des articulations temporo-mandibulaires. Le succès de cette technique non invasive, est lié à la mise en évidence pour la première fois in vivo de l'aspect statique et dynamique du disque articulaire. La masse de travaux publiés, plus de 200 dans les 10 dernières années, témoigne de l'engouement du monde odonto-stomatologique pour une technique qui lui permet de lever, sans douleur, le voile sur une structure profonde de la face. Mais il ne faut pas pour autant occulter les contre indications médicales qui sont exceptionnelles. En effet, les conséquences biologiques dues aux variations rapides du champ dans l'espace et le temps peuvent être dramatiques comme par exemple la fibrillation ventriculaire, sans compter l'échauffement, conséquence de l'effet des ondes hautes fréquences et de hautes puissances, qui peut être favorisé par les implants métalliques (pacemaker, prothèse métallique, clips... ). Le revers de la médaille est incontestablement le coût élevé des examens qui pourrait inciter les organismes sociaux à limiter le droit de prescription dans un avenir proche. Ceci est d'autant plus à craindre que I'IRM est une technique complexe caractérisée par la diversité des paramètres techniques et fondamentaux mis en œuvre et dont la connaissance n'est que peu répandue parmi les prescripteurs.

Cet article a pour but, à partir d'une revue exhaustive de la littérature, de fournir une explication sommaire mais exacte des principes de I'IRM, de présenter l'évolution des moyens d'investigations (matériels, dispositifs annexes), de préciser les choix des divers paramètres d'acquisition, de présenter les résultats cliniques obtenus sur des ATM normales ou pathologiques.

\section{COMPRENDRE L'IMAGERIE PAR RÉSONANCE MAGNÉTIQUE}

L'imagerie par résonance magnétique utilise le noyau (d'où l'épithète «nucléaire » qui lui était autrefois adjoint et que l'on a supprimé parce qu'il faisait peur !) d'hydrogène comme support de l'information. Celui-ci est en effet en forte densité dans les tissus humains $(75 \%)$ et il est par ailleurs le plus simple de ceux des noyaux à nombre impair de protons et neutrons pouvant présenter un magnétisme détectable (phosphore, carbone, sodium et surtout hydrogène).

\section{Le spin et la précession}

Le noyau d'hydrogène ne possède qu'un seul proton. II est animé d'un mouvement spontané de rotation sur lui-même appelé spin (rotation). En tournant sur elle-même, la charge positive du proton hydrogène induit un champ microscopique. Les noyaux d'hydrogène se comportent ainsi comme de véritables petits aimants et possèdent donc un moment magnétique $m$. A l'état naturel, la distribution et la direction des moments magnétiques sont réparties de façon aléatoire : leur résultante $M$ est nulle et le corps humain ne présente aucun magnétisme décelable. Par contre lorsque les moments magnétiques sont soumis à l'action d'un champ magnétique Bo très puissant, de 1000 à 15000 Gauss soient de 0.1 à 
1.5 Tesla (le champ magnétique terrestre est de 0.5 gauss), ils s'orientent selon la direction $d u$ champ magnétique Bo. En réalité, les moments magnétiques ne s'alignent pas strictement sur Bo, mais tournent autour de lui avec une angulation qui est proportionnelle à sa puissance. $\mathrm{Ce}$ mouvement de rotation autour du champ principal est comparable à celui d'une toupie : c'est la précession dont la vitesse est proportionnelle à I'intensité du champ principal. Un état d'équilibre est atteint (Fig. 1a et b).

\section{La résonance}

Arrivés à ce stade, les protons sont prêts pour la résonance, c'est à dire pour l'absorption puis la restitution d'une quantité d'énergie avec passage d'un niveau d'énergie à l'autre. Pour faire résonner les protons, on les soumet à un autre
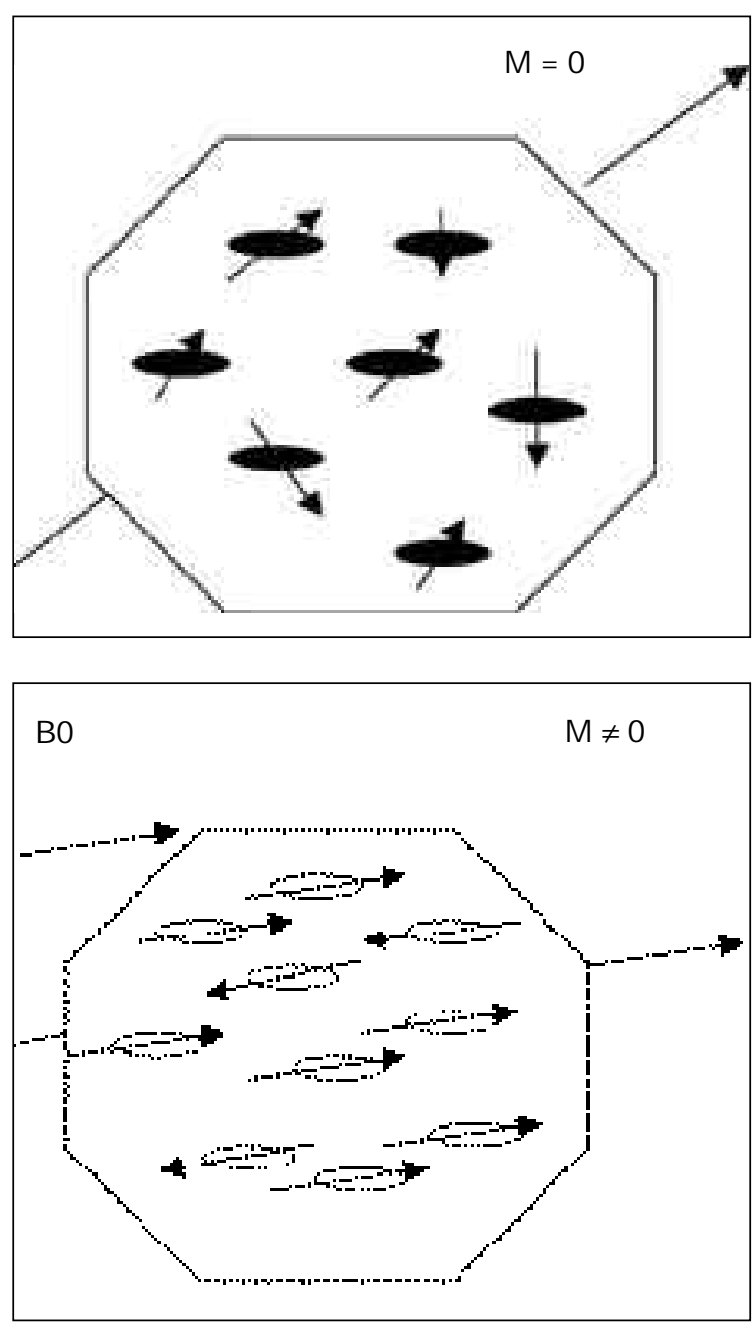

champ magnétique perpendiculaire au premier $B 1$. Ce deuxième champ B1 est créé grâce à une sonde de radio fréquence ou impulsion dont la nature vibratoire sinusoïdale équivaut à deux champs magnétiques tournant en sens inverse l'un de l'autre. Celui des deux champs B1 tournant dans le même sens que le mouvement de précession des protons entrera en résonance avec eux. B1 est beaucoup plus puissant que Bo, néanmoins il restera sans effet tant qu'il n'atteindra pas la vitesse de précession des protons. Lorsque $B 1$ atteint la vitesse de précession et une fréquence précise (dite de LARMOR), et ce pendant une durée suffisante, le moment résultant des protons se couche à $90^{\circ}$ et devient perpendiculaire à Bo. La bascule du moment résultant des protons sous l'effet de l'impulsion traduit un apport d'énergie, l'excitation (Fig 2a, b).

médecine buccale chirurgie buccale VOL. $8, \mathrm{~N}^{\circ}$ 2002 page 9

Figure 1 (a) : La distribution et la direction des moments magnétiques sont réparties de façon aléatoire en l'absence de champ magnétique.

Normally protons are aligned in a random fashion when no magnetic field is present.

Figure 1 (b) : Lorsque les moments magnétiques sont soumis à l'action d'un champ magnétique B0 très puissant, ils s'orientent selon la direction de ce champ.

Protons exposed to a strong external magnetic field B0. They align itselves in stability position in the magnetic field's direction. 

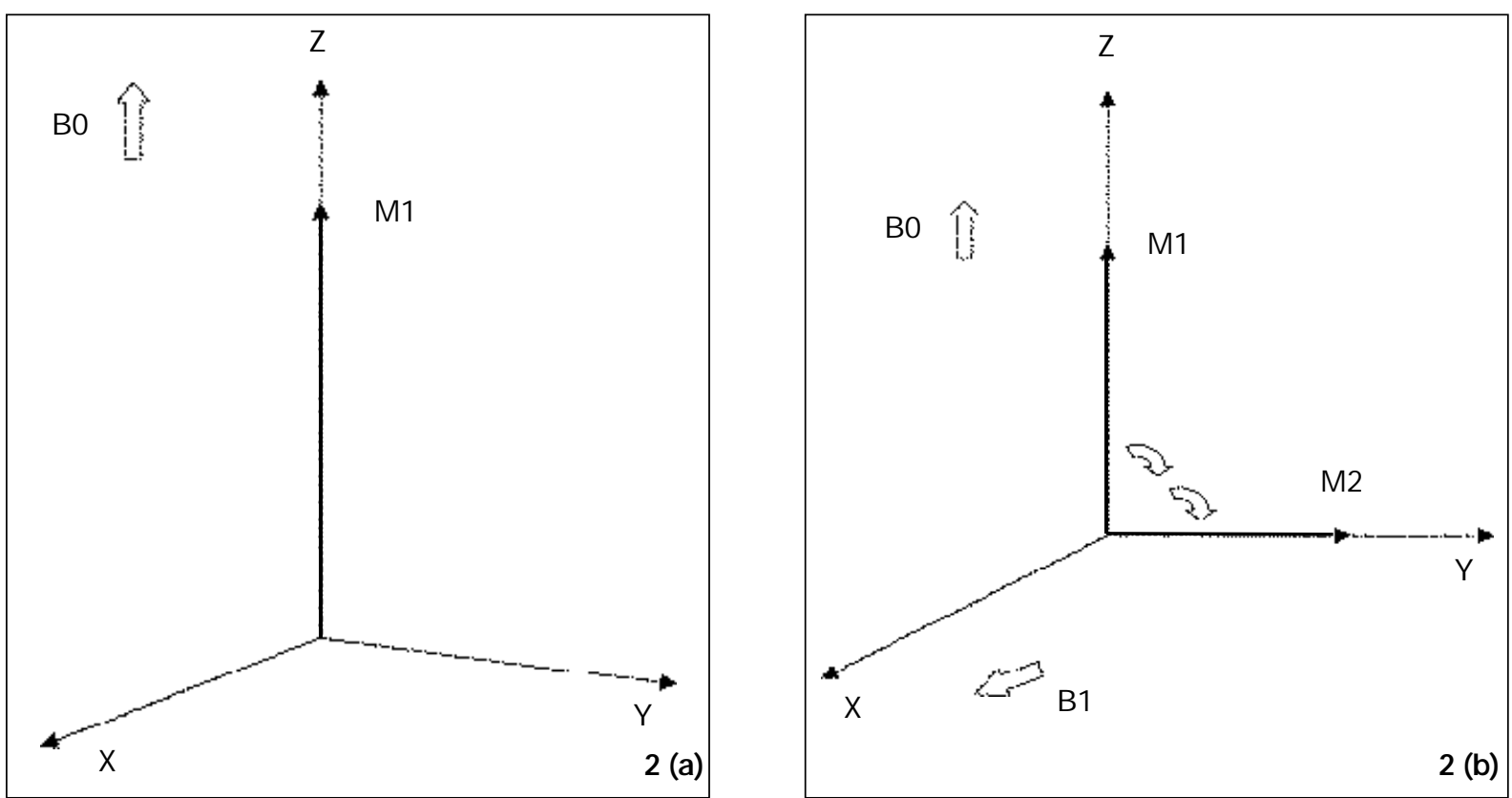

Figure 2 (a) : Situation du vecteur M1 avant l'impulsion RF

Situation before an RF pulse is sent in. Note the direction of the longitudinal vector M 1 parallel to $B 0$.

Figure 2 (b) : Situation après une impulsion $R F(B 1)$ de $90^{\circ}$ le vecteur $M 1$ subit un basculement de $90^{\circ}$ et devient M2 créant une magnétisation longitudinale.

M1 becoming M2 after the RF pulse which causes this longitudinal magnetization to decrease, and with a $90^{\circ}$ pulse as illustred it become zero.

\section{La relaxation et le signal}

A l'arrêt de l'impulsion, les protons excités, mis en cohérence de phase par l'onde de radio fréquence vont revenir à leur état initial d'équilibre défini par la présence du champ magnétique $\mathrm{B} o$. L'énergie captée pendant la phase d'excitation de la résonance est restituée sous forme d'onde de radiofréquence qui constitue le signal IRM. Celui-ci est mesuré par une antenne réceptrice et une chaîne d'amplification. Cette phase de retour à l'état initial est le temps de relaxation. Le temps de relaxation comprend deux composantes :

- le temps de relaxation longitudinal T1,

- le temps de relaxation transversal T2.

Le temps de relaxation longitudinal $\mathrm{T} 1$ est le temps de réalignement des protons. II correspond au temps nécessaire à la composante longitudinale du moment $M$ pour retrouver, après annulation, une valeur égale à $63 \%$ de sa valeur d'équilibre (Fig. 3 a). Ce temps est fonction de la densité en protons des tissus et il est également fonction de l'état pathologique : la richesse en eau d'une tumeur élève considérablement la valeur $\mathrm{T} 1$.

Le temps de relaxation transversal T2 est le temps de déphasage des protons entre eux c'est à dire le temps de perte de leur cohérence de phase créée par le champ B1. En pratique, il représente le temps mis par l'aimantation transversale $\mathrm{Mt}$ composante transversale du moment $M$, pour retrouver après son annulation une valeur égale à $37 \%$ de sa valeur initiale. (Fig. 3b). Cette désynchronisation varie en fonction des composantes tissulaires. T2 est long pour les liquides (200 à 600 ms) comme le liquide céphalo-rachidien, court pour les solides comme l'os.

On conçoit dès lors que le contraste naturel de l'image va dépendre à la fois de la densité protonique des tissus explorés et aussi de la variation des temps de relaxation des différents composants tissulaires, aussi bien pour T1 que pour T2. L'étude des vitesses de relaxation des différents tissus permet ainsi d'identifier au cours d'une séquence le moment idéal pour recueillir le signal avec une qualité de contraste maximum. 


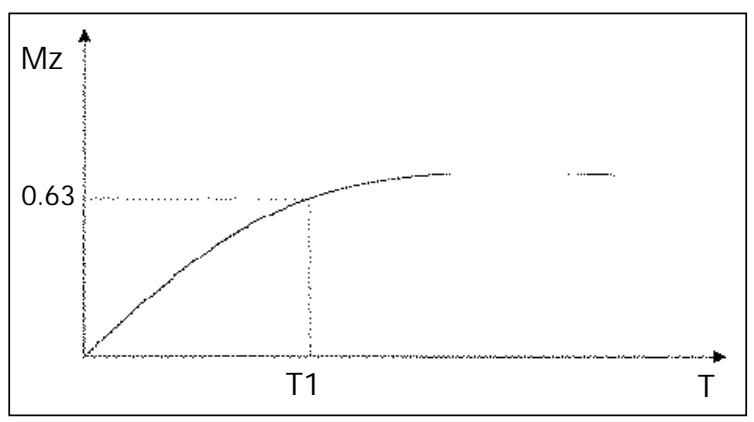

Figure 3 (a) : Courbe T1

T1 curve

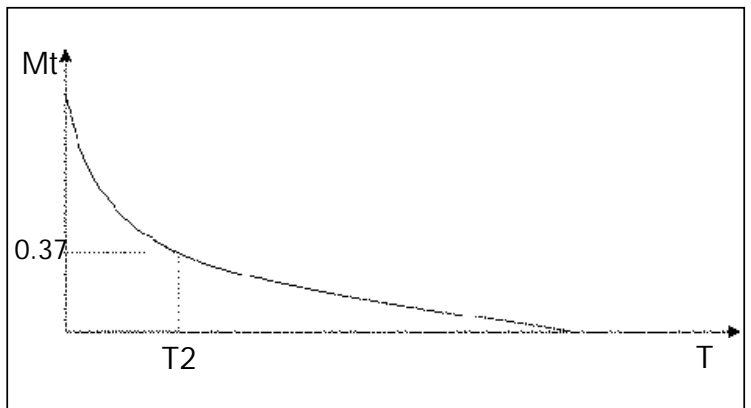

Figure 3 (b) : Courbe T2

T2 curve

\section{L'image obtenue}

Trois paramètres concourent au résultat de l'exploration IRM :

- la densité protonique $\rho$,

- le temps de relaxation $T 1$,

- le temps de relaxation $\mathrm{T} 2$.

Chaque tissu est en réalité caractérisé par un T1 et un T2 spécifiques qui traduisent sa composition moléculaire. Les variations de $\mathrm{T} 1$ et $\mathrm{T} 2$ sont ainsi liées soit au passage d'un tissu à l'autre soit au changement physiopathologique à l'intérieur d'un tissu donné (phénomènes inflammatoires en particulier). Ces temps de relaxation sont appréciés en millisecondes.

Mais, compte tenu du fait qu'un examen et donc la production du signal IRM est le résultat non pas d'une seule, mais d'une série d'impulsions successives (on dit une séquence), deux autres facteurs vont permettre de moduler la reconstruction de l'image. Ce sont le temps de répétition (TR) et le temps d'écho (TE).

Le temps de répétition TR est le temps qui sépare le début d'une séquence du début de la séquence suivante. Le temps d'écho TE est, à l'intérieur d'une même séquence, le temps qui sépare l'impulsion initiale de l'obtention de l'écho : lors de la première impulsion nous avons vu que l'aimantation résultante amène le moment $\mathrm{M}$ à $90^{\circ}$ de $\mathrm{Bo}$; cette impulsion provoque l'émission d'un signal qui n'est pas directement utilisé ; la deuxième impulsion amène $M$ en sens inverse, à $180^{\circ}$ et provoque l'émission d'un deuxième signal qui renvoie le premier comme un mur. C'est l'écho qui lui, est à l'origine de l'image. Les temps TR et TE sont réglables par l'opérateur et permettent ainsi de moduler les valeurs relatives de $\rho, T 1$ et $T 2$ dans l'image résultante. Lorsque le TR et le TE sont courts (respectivement environ $500 \mathrm{~ms}$ et $20 \mathrm{~ms}$ ) l'image est très pondérée en T1. Lorsque le TR et le TE sont longs (respectivement environ 2000 $\mathrm{ms}$ et $100 \mathrm{~ms}$ ) l'image est très pondérée $T 2$. En pratique, $\mathrm{T} 1$ et $\mathrm{T} 2$ sont donc les éléments essentiels dans la caractérisation de l'image permettant de faire apparaître les tissus sur l'image en hyper signal blanc (pour la graisse par exemple) ou en hyposignal noir (pour le disque articulaire pauvre en eau). Néanmoins, pour certaines valeurs de TE et TR, l'influence de $\mathrm{T} 1$ et $\mathrm{T} 2$ diminue et c'est la densité protonique $\rho$ qui conditionne la nature de l'image. On parle alors d'images en densité protonique qui sont généralement peu contrastées ( $T R=1500 \mathrm{~ms}, T E=30 \mathrm{~ms}$ ). L'image obtenue peut enfin être modifiée par l'utilisation d'une injection intraveineuse de Gadolinium di-éthylène triamine penta acétate (Gd DTPA). Le but de ce produit paramagnétique est d'accroître le champ local grâce à l'aimantation engendrée (1800 fois supérieure à l'aimantation nucléaire). Celle-ci entraîne une accélération de la relaxation au cours d'une séquence pondérée en $\mathrm{T} 1$ et permet ainsi d'observer l'étendue précise et les limites d'une lésion inflammatoire ou tumorale en proportion du flux hématique qui y circule. Nous verrons, appliqué à l'exemple particulier des articulations temporo-mandibulaires, à quelles images aboutit la manipulation de ces divers paramètres. Pour résumer, on peut dire que la pondération $\mathrm{T} 1$ fournit une image aux contours anatomiques précis tandis que la pondération T2 permet la mise en évidence marquée des phénomènes inflammatoires associés. médecine buccale chirurgie buccale VOL. $8, \mathrm{~N}^{\circ}$ 2002 page 11 
médecine buccale chirurgie buccale

)L. $8, \mathrm{~N}^{\circ} 1$

2002

page 12

\section{LES NÉCESSITÉS TECHNOLOGIQUES}

Elles comportent d'une part la sélection d'un certain nombre d'équipements, c'est l'appareillage ; d'autre part, à équipement égal, le choix de paramètres spécifiques. L'ensemble de ces nécessités technologiques conditionne l'image obtenue et son interprétation statique et dynamique.

\section{L'appareillage}

La chaîne qui permet d'aller de la structure anatomique à l'image IRM comprend donc, d'une façon générale: des aimants qui créent les divers champs magnétiques, des antennes qui récupèrent le signal IRM, un lourd dispositif informatique qui convertit le signal en image.

Nous allons voir en ce qui concerne les deux premiers éléments de cette chaîne, les caractéristiques spécifiques de l'exploration des ATM. II nous faut y ajouter les différents dispositifs d'ouverture buccale qui sont indispensables à l'exploration dynamique de cette articulation.

\section{Les aimants}

Le champ magnétique principal Bo idéal est un champ homogène et stable dans le temps. Un tel champ est créé par des aimants supraconducteurs. La puissance de Bo peut dépasser 2 Tesla. J usqu'à ces dix dernières années, différents imageurs de puissances allant de 0.1 à 2 Tesla ont été utilisés dans l'exploration de I'ATM. Les imageurs à hauts champs représentent la grande majorité : $68 \%$ des auteurs ont utilisé des imageurs de $1.5 \mathrm{~T}$ pour l'exploration des ATM. Cette valeur est-elle la mieux indiquée pour l'exploration de l'ATM ou résulte-t-elle d'une convergence fortuite? Les bases théoriques concernant la relation entre le champ Bo et le rapport signal sur bruit (S/B) montrent qu'à $1.5 \mathrm{~T}$ ce rapport est trois fois plus important qu'à 0.5 . Hanson et coll [5] dans une étude comparative entre deux valeurs de champs $0.3 \mathrm{~T}$ et 1.5 T, ont mis en évidence l'avantage d'un haut champ dans l'optimisation des séquences. Néanmoins ces résultats obtenus sur des coupes de cadavre frais congelé ne semblent pas transposables tels quels à l'examen in vivo.
Les antennes (Fig. 4 a, b)

L'antenne constitue l'un des éléments essentiels de la chaîne radiofréquence, tant à l'émission qu'à la réception. Ses performances dépendent de sa taille, de sa forme et de la distance qui la sépare de la région explorée. II existe par conséquent :

- des antennes en selle qui émettent un champ $R F(B 1)$ très homogène et dont la dimension est adaptée à la région explorée, les antennes selles sont utilisées comme bobines émettrice et réceptrice et équipent tous les imageurs ;

- des antennes à polarisation circulaire qui permettent de diviser par deux la puissance absorbée par le patient et d'améliorer encore l'homogénéité de B1 et la sensibilité ;

- l'antenne de surface dont la sensibilité diminue avec la distance à la surface, ce qui limite le volume détecté à une demi-sphère de diamètre égal à celui de l'antenne interdisant de ce fait l'exploration des organes profonds.

L'antenne de surface a cependant des avantages qui en font un élément essentiel dans l'exploration de l'ATM [6-12] :

- elle s'adapte à la région explorée [13];

- elle fournit un meilleur rapport $S / B$, nécessaire à une haute résolution spatiale et à la réduction du temps de mesure, autrement dit du temps d'examen ;

- elle se montre particulièrement utile dans les séquences rapides qui se caractérisent par des signaux de faible intensité et un niveau de bruit élevé.

Hyde et coll ${ }^{[14]}$ semblent cependant apporter une amélioration du rapport S/B grâce à une antenne de configuration originale, appelée «PLANAR-PAIR». L'une de ses caractéristiques est son indépendance par rapport à l'antenne «corps» de l'imageur (Fig. 5).

D'autres auteurs ont décrit des systèmes d'antenne double ou bobine bilatérale pour l'étude des ATM : Hardy et coll [15], Wright [16], Shellholk [17]. La bobine bilatérale permet, un examen des deux ATM au même degré d'ouverture buccale. Les deux mouvements de translation opérés par les condyles peuvent être alors évalués et comparés. Grâce à la prise d'images à différents degrés d'ouverture buc- 
cale en mode pseudo cinématique, non seulement, on enregistre le mouvement du complexe disco-condylien, mais également la rotation de la tête mandibulaire par rapport au disque [18].

\section{Les dispositifs d'ouverture buccale et leur influence sur le degré optimal d'ou- verture buccale}

L'exploration idéale d'une articulation comporte l'examen de ses structures fixes et surtout de ses structures mobiles, en mouvement. Dans le cas particulier de I'ATM, seul le mouvement d'ouverture-fermeture est régulièrement exploré parmi ses degrés de liberté anatomiques. Néanmoins, sachant que tout mouvement du patient au cours de l'examen IRM fausse consi-

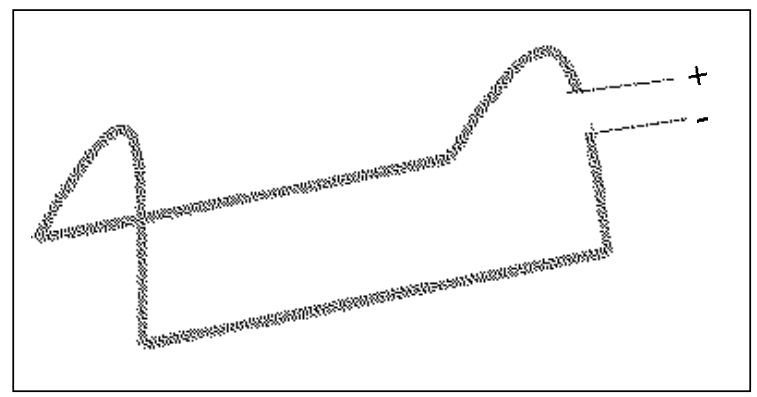

Figure 4 (a) : Antenne en «selle» Saddle coil

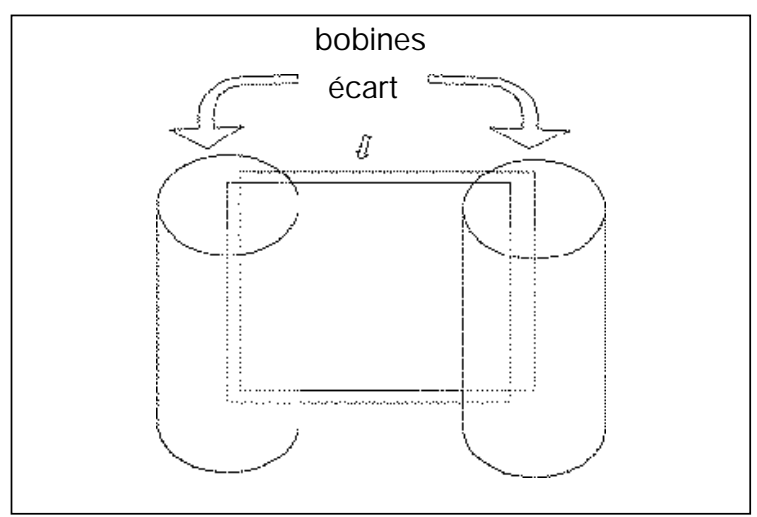

Figure 5 : Antenne Planar-Pair d'après Hyde

- Les bobines forment l'inductance

- L'écart fournit la capacitance

Planar-Pair loop-gap resonator:

- the loops form the inductance

- the gap provides capacitance dérablement le recueil du signal, le mouvement d'ouverture fermeture ne peut être examiné que comme une succession de paliers fixes. C'est en soi une limite fondamentale de l'examen que l'odontologiste doit avoir à l'esprit lors de l'interprétation des images.

Burnett et coll [19] a mis au point un dispositif d'ouverture buccale par paliers progressifs, activable par le patient, mettant ainsi en évidence, en mode pseudo cinétique, le mouvement de translation du complexe disco-condylien (Fig. 6).

En phase dynamique, certaines transformations pathologiques et particulièrement la phase de déformation du disque lors de la subluxation chez les patients présentant des dysfonctions

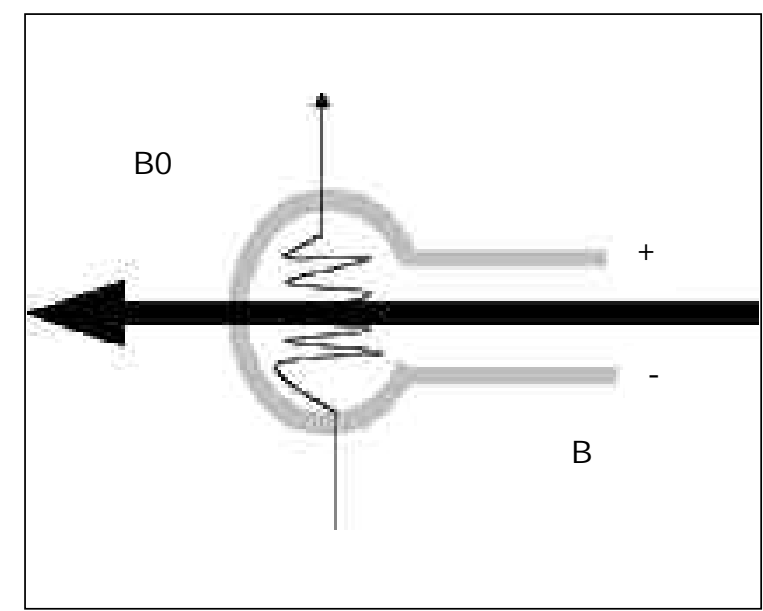

Figure 4 (b) : Antenne de surface Surface coil

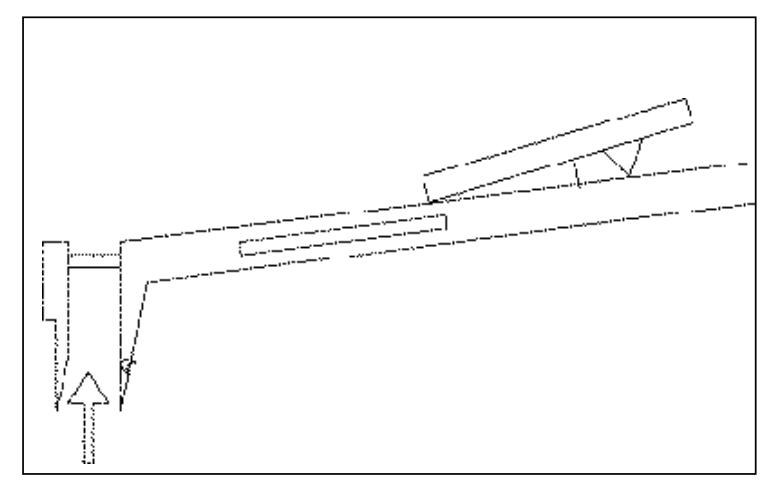

Figure 6 : Appareil de Burnett Burnett device médecine buccale chirurgie buccale VOL. $8, \mathrm{~N}^{\circ}$ 2002 page 13 
médecine buccale chirurgie buccale

)L. $8, \mathrm{~N}^{\circ} 1$ 2002 page 14 de I'ATM, ont été étudiées en mode cinématique par le moyen de cales d'ouverture [20]. Ce mouvement passif de la mandibule appelle quelques réserves, car il ne peut se substituer à un mouvement naturel, libre de tout dispositif d'ouverture buccale où les signes de dysfonctions mandibulaires seraient perçus sans entraves. Un autre dispositif d'ouverture mis au point par Vogl et coll [21] fait appel au mode hydraulique (Fig. 7). Grâce à un piston, des positions significatives d'ouverture et de fermeture sont localisées d'une façon précise lors d'un examen. Ces dispositifs doivent permettre d'obtenir une phase optimale et stable d'ouverture buccale. En effet, l'exploration de l'ATM et de ses structures anatomiques ne peut se faire que dans les positions bouche ouverte (BO), bouche semi-ouverte $(B 1 / 20)$, bouche fermée (BF) $[14,22,23]$.

Cependant, la position buccale de départ, pour une exploration de l'ATM en mode cinématique, est une position BF en l'absence de tout dispositif buccal. La méconnaissance de ce point peut être la cause d'une erreur d'interprétation de la position du disque articulaire au départ du mouvement mandibulaire [24].

Drace et coll $[25,26]$ ont tracé les histogrammes de distribution des positions discales BF et B1/2O (Fig. 8). Ces positions sont considérées par rapport à une position standard, la position de midi que Katzberg et coll [27] ont définie. A partir de là, est considéré positif tout déplacement antérieur et négatif, tout déplacement postérieur. La conclusion de Drace et coll $[25,26]$ est que seule la position BF met en évidence la position exacte du disque. Cette étude ne fait pas I'unanimité. Sanchez-Woodworth et coll [28] recommandent, l'examen systématique des deux ATM, BO et BF, chez les patients qui présentent des signes de dysfonctionnement d'une seule ATM.

Pour Orwig et coll [29] la position BF améliore la précision du diagnostic dans la pathologie du déplacement discal, mais la position B1/20, permet de desserrer les structures de l'ATM et d'obtenir une vue optimale du disque. (intensité de son signal et sa morphologie).

\section{Le choix des paramètres}

Le choix porte ici d'une part, sur les paramètres de localisation qui comportent deux éléments essentiels : l'épaisseur de coupe et le plan de coupe; d'autre part sur des éléments dont nous avons vu le lien direct avec la composition tissulaire dans la présentation des principes.

\section{Les paramètres de localisation}

\section{- Le volume du voxel}

On appelle voxel le plus petit volume élémentaire d'une tranche d'organe qu'on excite en IRM. Le volume du voxel choisi est la conséquence d'un triple choix : le choix du champ d'analyse (Field of View) (FOV), le choix de la matrice d'acquisition et enfin le choix de l'épaisseur de coupes.

Choix du FOV : ce paramètre est fonction de la taille de l'antenne utilisée et de celle de la région à analyser. La plupart des auteurs réalisent leurs examens à partir d'antennes de surface de $6 \mathrm{~cm}$ à $18 \mathrm{~cm}$ de diamètre.

Choix de la matrice d'acquisition : elle est habituellement de $256 \times 256$. Son choix, semble lié à l'expérience de l'utilisateur et non à des normes scientifiquement établies.

Choix de l'épaisseur de coupe : d'une façon générale, diminuer l'épaisseur des coupes permet de restreindre l'effet du volume partiel mais ceci ne se fait qu'au prix d'une diminution proportionnelle du rapport S/B et d'une diminution de l'épaisseur globale analysée (à nombre constant de coupes). La réalisation de coupes en 3 DFT permet des coupes fines $(1 \mathrm{~mm}$ si besoin) et jointives. En contre partie, elle nécessite un temps d'exploration et de reconstruction prohibitif. C'est pourquoi, même si la qualité de l'image est inversement proportionnelle à l'épaisseur de coupe, la valeur la plus fréquemment retenue est de $3 \mathrm{~mm}$. [30,31]

\section{- Les acquisitions 2 DFT et 3 DFT dans l'exa- men de l'ATM}

Nous savons que l'acquisition 2 DFT est une méthode qui permet des acquisitions plan par plan tandis que la technique 3 DFT permet l'acquisition d'un volume entier. Concernant l'ATM l'acquisition $3 \mathrm{D}$ présente des avantages : 
visualisation de coupes très fines (et donc d'un nombre de coupes plus élevé), visualisation de coupes jointives, amélioration du rapport S/B. Mais, les inconvénients tels que l'augmentation des artéfacts de mouvements et l'augmentation de la durée d'acquisition relativisent ces avantages. Ainsi une sensibilité accrue à l'inhomogénéité du champ magnétique des acquisitions $3 \mathrm{D}$, leurs limites quant aux altérations physiologiques des structures intra-articulaires des muscles manducateurs, et de la moelle osseuse, font qu'elles ne sont pas les mieux indiquées pour visualiser I'ATM et ses structures anatomiques. [32].

\section{- Les plans de coupes}

Le choix du plan de coupe est essentiel à l'identification en IRM d'une des structures composants l'ATM. Ce que les anglo-saxons appellent Région Of Interest (ROI).

Le premier plan utilisé pour l'exploration de I'ATM fut le plan sagittal. Les coupes sagittales ont permis à Helms et coll [4] d'explorer l'ATM d'un point de vue strictement anatomique. Le plan sagittal permet de décrire la position discale dans le sens ventro-dorsal [33-35]. II permet également de détecter les anomalies de surface au niveau de la surface articulaire de la tête mandibulaire [36].

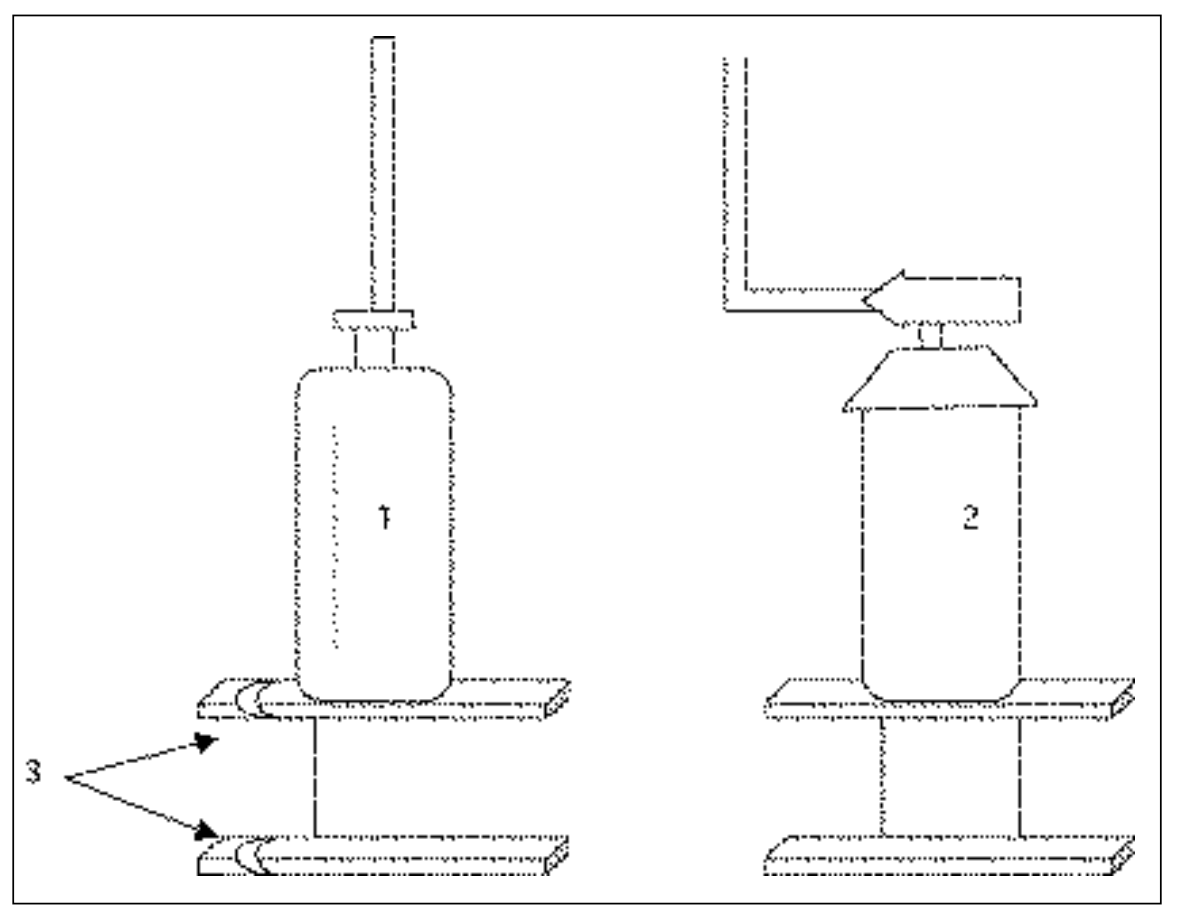

Figure 7 :

Appareil hydraulique de Vogl

Comportant 2 pistons dont un $\left(n^{\circ} 1\right)$ comporte des indentations (3), le $\mathrm{n}^{\circ} 2$ est gradué de façon à mesurer l'ouverture buccale du sujet.

Vogl hydraulic device contains: a first piston $n^{\circ} 1$ with indentation (3), a second piston $\mathrm{n}^{\circ} 2$ with screw thread to calibrate the jaw opener for MR imaging of TMJ .
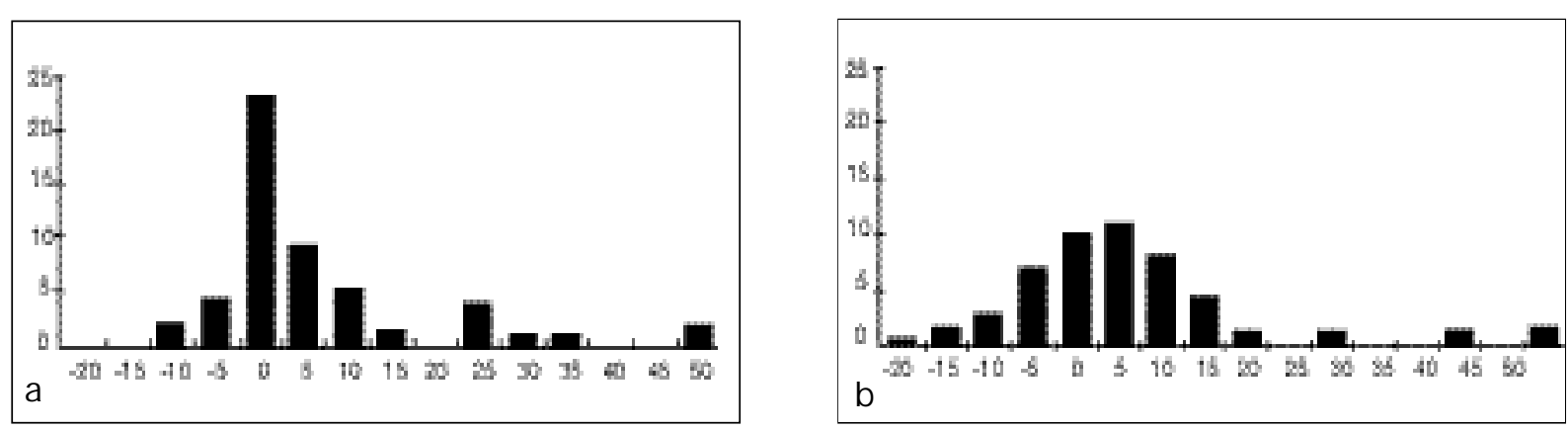

Figure 8 : Histogrammes de distribution des positions méniscales BF (a) et B 1/2 O (b) selon Drace Distributions of disk positions in both closed and half open mouth positions according to Drace 
médecine buccale chirurgie buccale
En 1988, Katzberg et coll [37] suivi par la plupart des autres auteurs [32,38-40] introduisent les coupes coronales. Ces auteurs prétendent visualiser des déplacements médio-latéraux du disque qui ne sont pas rares ainsi que leurs attaches médiales et latérales. Cette suggestion pourrait s'insérer dans l'examen de routine si l'interprétation de ces coupes était aisée. Or l'expérience montre que ce n'est pas le cas et de nombreux radiologues ne les pratiquent pas. Musgrave et coll [41] préconisent un plan parasagittal perpendiculaire au grand axe de la tête mandibulaire et un plan para-coronal parallèle à ce grand axe. Ces orientations améliorent la visualisation des tissus mous, en particulier le disque et la relation disco-ptérygoïdienne. Ceci fut confirmé par Tasaki et coll [42]. Mais la vogue de ce plan de coupe est probablement en décrue car les déplacements de l'ATM ne se font pas dans ce plan mais bien dans un plan sagittal.

Des plans standards ont finalement été définis afin de visualiser les structures anatomiques de I'ATM. Une coupe axiale de localisation permet de repérer l'axe de la tête mandibulaire. Des coupes para sagittales et sagittales permettent d'identifier les différentes structures de l'ATM [43]. Plus récemment, Matsuda $S$, et Yoshimura [44], ont préconisé la combinaison des deux plans sagittal et coronal pour la visualisation du déplacement discal dans les luxations. II faut souligner que l'immobilisation de la tête du patient à l'aide d'un céphalostat minimise les risques d'artéfacts et facilite la comparaison des plans de coupes avant et après traitement par exemple. [12,45-48]. Cette méthode très approximative devrait être remplacée rapidement par des méthodes de recalage mathématique dans un céphalostat virtuel. [49]

\section{- Choix des séquences}

Rappelons qu'une séquence est une succession d'impulsions d'ondes de radio fréquence se produisant à des intervalles de temps variables.Le choix des séquences dépend essentiellement du contraste que l'on veut obtenir.

- Les séquences en écho de spin (SE)
La séquence SE se compose de 2 impulsions $90^{\circ}$ et $180^{\circ}$. Deux intervalles de temps la caractérisent, le TR et le TE. Après l'impulsion de $90^{\circ}$, les protons sont en phase. II y a ensuite perte progressive de cohérence de phase car certains protons tournent plus vite que d'autres.

L'écho de spin est la séquence la plus utilisée. Elle permet la mise en évidence de toutes les structures de l'ATM d'un point de vue tant physiologique que pathologique. Cette séquence, dépend de certains paramètres liés aux différents tissus composant I'ATM (N), T1 et T2. La qualité de l'image résulte donc de la valeur de ces différents paramètres :

- si TE croît par rapport à T2, l'intensité I du signal décroît progressivement,

- si TR croît par rapport à T1, l'intensité I du signal augmente.

Semmler $W^{[50]}$ a proposé des paramètres standards pour des séquences SE pour un champ magnétique de 2 Tesla :

- pour des séquences en $N(H)$ : TR>1600 et $\mathrm{RE}<35 \mathrm{~mm}$;

- pour des séquences en T1:TR $<400$ et $T E<35$;

- pour des séquences en T2 : TR>1600 et $\mathrm{TE}>70$;

- Intérêt et limite des séquences en Echo de Gradient (EG).

Ces séquences sont dites "d'imageries rapides» car elles permettent de diviser par 10 le temps d'acquisition. Contrairement à la séquence $S E$ qui crée un écho grâce à une impulsion radio fréquence de $180^{\circ}$, la séquence EG génère un écho par une astuce technologique : I'inversion du gradient de lecture. On décrit alors 3 paramètres de machines TR, TE et $\alpha^{\circ}$ (angle de basculement du vecteur $M$ qui représente la résultante des protons du tissu considéré). Grâce à la réduction du TA [51-53] cette séquence qui ne comporte qu'une impulsion unique a permis la réalisation du mouvement de l'ATM en mode pseudocinématique [19-20]. Cet avantage n'est cependant pas sans réserves car, la diminution du TA s'accompagne souvent d'une visualisation médiocre des structures essentielles, telles que le disque [54]. Elle est également à l'origine de diagnostics erronés à cause des artéfacts, créés par l'artère tempo- 
rale superficielle, et de ses différents aspects dus aux manifestations du flux sanguin lors des mouvements capitaux [55]. De plus les séquences rapides, ne peuvent montrer les remodelages dégénératifs du cartilage ou des articulations arthroscopiques, car elles donnent moins de détails anatomiques que les séquences $S E$ du fait du phénomène de saturation [56]. Schellhas et coll [57-62] recommandent d'utiliser les deux types de séquences pour évaluer les symptômes douloureux des ATM avec ou sans symptômes mécaniques, tels que le craquement ou le claquement.

\section{ANATOMIE IRM STATIQUE DE L'ATM NORMALE}

\section{L'ATM proprement dite}

Nous allons décrire ici le signal des différents constituants de l'ATM en prenant en compte la pondération $\mathrm{T} 1$ et le plan sagittal de loin le plus explicite sur le plan anatomique (Fig. 9)

\section{La tête mandibulaire}

La tête mandibulaire se manifeste par un hypersignal, correspondant à l'os spongieux où siège la moelle, bordé d'un hyposignal correspondant à la corticale. Sur un examen pratiqué en direction latéro-médiale, la tête apparaît seule sur les coupes les plus latérales; elle est ensuite prolongée en ventro-caudal par le col de la mandibule et progressivement des éléments de la branche.

\section{La surface articulaire temporale}

Le tubercule articulaire est matérialisé par une image caractéristique d'hypersignal bordé par un hyposignal sur les coupes centrales de l'articulation. La fosse mandibulaire apparaît comme une ligne d'hyposignal fine parfois confondue avec la masse du diploë.

\section{Le disque articulaire}

On sait $[4,14,22,63,64]$ que le disque dans son état normal émet un hyposignal quelque soit la séquence utilisée, car il est constitué de fibrocollagène. Ce signal, bien que de faible intensité, est souvent bien distinct compte tenu des signaux les plus intenses des tissus environnants. Le contraste entre les différentes structures augmente avec le TR de la séquence utilisée. En effet, les signaux émis par les différents tissus composants l'ATM, ont été définis par des séquences SE à TR et TE courts.

Il en résulte que :

- la graisse (y compris celle présente à l'intérieur du col de la mandibule) donne un hypersignal dû à son court temps de relaxation $\mathrm{T}$;

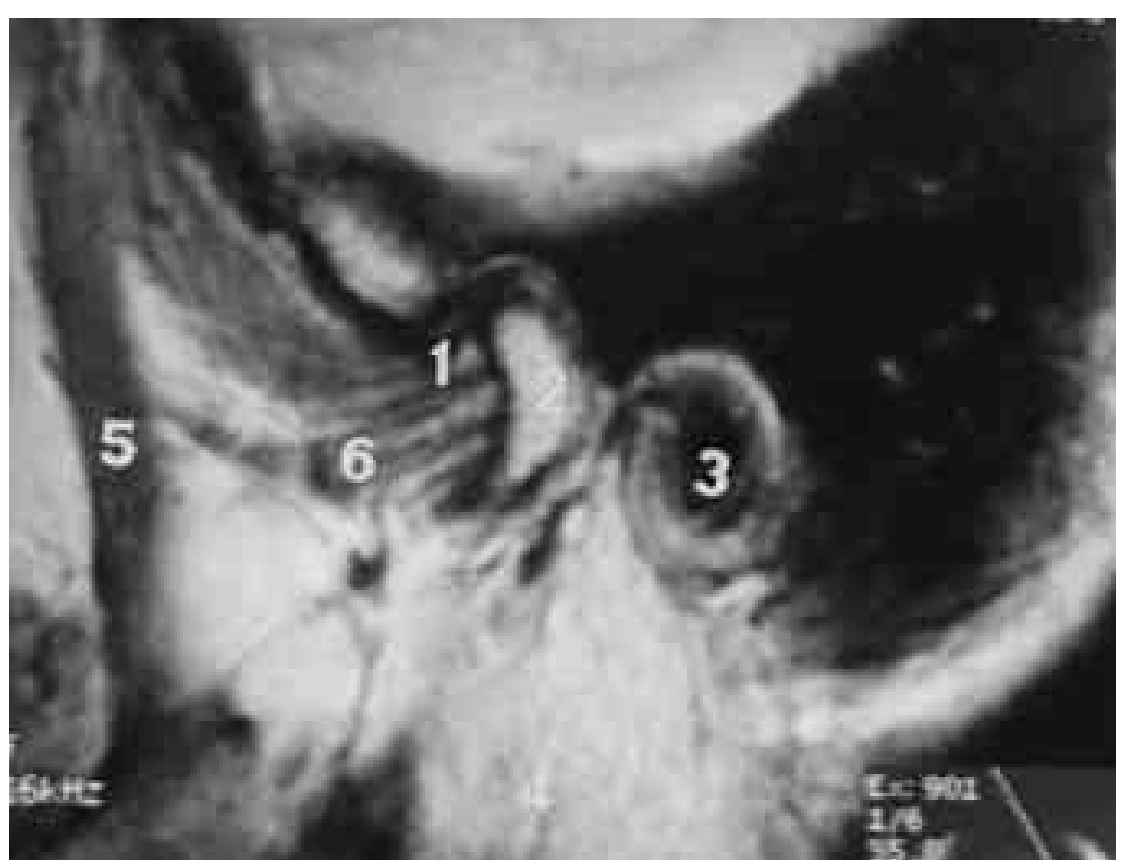

Figure 9 : Image caractéristique de coupe sagittale en IRM. Noter les différents signaux des structures anatomiques visibles : le disque (1) le condyle (2) - conduit auditif externe (3) - parotide (4) carotide externe (5) - muscle ptérygoïdien latéral (6).

A sagittal resonance image of TMJ. We note the different components of this articulation: the disk (1) - the mandibular condyle (2) - external auditory canal (3) - parotid gland (4) - external carotid (5) lateral pterygoid muscle (6). médecine buccale chirurgie buccale VOL. $8, \mathrm{~N}^{\circ}$ 2002 page 17 
médecine buccale chirurgie buccale

)L. $8, \mathrm{~N}^{\circ} 1$

2002

page 18
- la corticale donne un hyposignal dû à une teneur en eau très faible ;

- le muscle présente un signal intermédiaire entre celui de la graisse et celui de l'os cortical ;

- le cartilage articulaire hyalin à un signal d'intensité intermédiaire, entre celui du muscle et celui de la graisse ;

- le disque présente un hyposignal.

Bien que structurellement proches, disque et cartilage, peuvent être différenciés grâce aux différents type de collagènes (type 1 et type 2), et à leur proportion respective dans chaque tissu. En effet, la quantité d'hydroxylyne est moins élevée dans le cartilage fibreux que dans le cartilage hyalin. Le collagène de type 1 est moins hydrophile que le collagène de type 2 . La quantité de protons est par conséquent moindre; le signal du cartilage hyalin a donc une intensité plus élevée que celle du cartilage fibreux contenu dans le disque [65,66]. Quant aux structures fines composant le disque luimême, plusieurs travaux concordants définissent leurs signaux en particulier pour la zone postérieure $[25,38,67]$. C'est ainsi que :

- la jonction entre le bourrelet dorsal et la zone bi laminaire émet un hyposignal ;

- le frein discal dorsal donne un signal plus intense que celui émis par le bourrelet dorsal, du fait de sa richesse en tissu adipeux ;

- la partie temporale du frein discal dorsal ne peut être visualisée que par les séquences SE à TR et TE courts à cause de la teneur en graisse très variable selon la puissance du champ Bo.

En résumé, le disque articulaire visualisé par I'IRM a l'aspect classique d'une section de lentille biconcave : son rebord dorso-crânial est situé au-dessus de la tête mandibulaire et son rebord ventro-caudal est situé en dessous du tubercule articulaire du temporal.

La présence de quelques gros vaisseaux peut être visible grâce à un signal très bas à cause du flux sanguins [68].

Les bourrelets ventral et dorsal ont un signal faible, plus noir, que la partie intermédiaire du disque, plutôt grise. Le bourrelet ventral est une structure homogène de section triangulaire. Le sommet de ce triangle est dorsal et se prolonge par la partie intermédiaire du disque. La base de ce triangle est ventrale et représente la limite ventrale de la capsule. Parfois, l'insertion du chef crânial du muscle ptérygoïdien latéral sur le bourrelet ventral est visualisée comme un signal de faible intensité, d'aspect filiforme et fixée au contour médio-ventral du disque.

Ce repère est moins constant que la démarcation toujours précise entre le bourrelet dorsal et la zone bilaminaire. Le bourrelet dorsal est triangulaire à sommet ventral. Sa base établit la jonction avec la zone bilaminaire. La partie centrale du bourrelet dorsal a un signal d'intensité plus élevée que la moyenne du disque. La limite dorsale du bourrelet dorsal est marquée par une ligne légèrement plus épaisse, orientée verticalement, de signal faible. La présence au centre du bourrelet dorsal d'une région de haute intensité correspond selon Katzberg et coll [75], à un dépôt de mucine sans signification clinique.

\section{Le tissu rétro discal}

Le tissu rétro discal a un signal d'intensité moyenne (signal intermédiaire iso intense). Il est situé dorsalement à la tête mandibulaire et ventralement au conduit auditif externe dont il est séparé par l'os tympanal. En général, il y a un bon contraste entre le bourrelet dorsal et la zone apparente lors de la translation ventrale de la tête mandibulaire.

La zone bilaminaire est identifiée par deux lignes horizontales très fines de faible intensité autour du centre du signal de haute intensité. Une petite structure linéaire avec un signal de faible intensité est parfois notée dans la zone bilaminaire dont elle semble représenter les fibres élastiques de la composante temporale.

\section{La musculature mobilisatrice}

La musculature mobilisatrice de I'ATM a fait l'objet d'une étude précise tant du point de vue anatomique que du point de vue mécanique. [76,77]. En IRM, la visualisation de la musculature oro-faciale est liée au degré d'ouverture buccale, [78-80]. L'acquisition 3 DFT, a servi à la localisation d'électrodes d'enregistrement en électromyographie [81].

Le muscle ptérygoïdien latéral a un signal d'intensité moyenne (gris). Le chef caudal se fixe 
directement sur la fossette ptérygoïdienne du col mandibulaire. Les deux chefs, caudal et crânial, sont bien individualisés par le tissu adipeux entourant le muscle. Ils sont de plus faciles à distinguer compte tenu de l'orientation différente de leurs fibres; ils sont enfin séparés par une bande de signal de faible intensité qui traduit la présence d'une lame aponévrotique. Par contre, les insertions capsulo-discales sont difficiles à discerner. Le corps musculaire a un signal homogène de sorte que la séparation des fibres musculaires est peu nette.

Le muscle temporal apparaît comme une structure d'intensité moyenne dont les fibres sont disposées verticalement ventro-crânialement au tubercule articulaire du temporal. Sur les coupes sagittales les plus médiales, son signal se résume à une simple bande qui correspond probablement à l'insertion profonde du faisceau orbitaire. Le muscle masséter est visible sur les coupes sagittales les plus latérales. II est alors situé caudalement au muscle temporal d ont il est séparé par l'hyposignal du processus coronoïde. La fasciculation de ce muscle est parfaitement lisible sur ces coupes et l'orientation différente des fibres permet également de le distinguer du muscle temporal. Seltzer et coll [82] constatent que le signal émis par le masséter dans les séquences à TR et TE courts est plus intense que dans d'autres séquences car une couche de graisse de T1 court entoure ce muscle.

\section{Les éléments de voisinages}

\section{Le processus coronoïde}

II n'est visible que sur les coupes les plus latérales. C'est un hyposignal dépourvu de frange d'hyper signal compte tenu de son immersion constante dans la masse des signaux intermédiaires correspondant aux fibres musculaires du temporal, du masséter, du buccinateur.

\section{La glande parotide}

Elle représente une masse importante sur les coupes les plus latérales. C'est un hyper signal très blanc situé dorso-caudalement à la tête mandibulaire. Des zones inhomogènes d'hyposignal sombre marquent la présence du système circulatoire carotidien et jugulaire.

\section{Le méat acoustique externe}

Il est dorsal par rapport à la tête mandibulaire et crânial par rapport à la masse parotidienne. Un hyposignal ovale très noir est entouré par un signal plus intense inhomogène, du gris au blanc, qui correspond aux cartilages du pavillon. Ce halo disparaît sur les coupes les plus médiales.

\section{La masse cérébrale}

C'est un signal intermédiaire gris et clair. Les circonvolutions sont nettement identifiées dans la partie crâniale de l'image. Le sinus latéral est toujours visible.

Nous venons de voir que les signaux émis par les différentes structures présentent des nuances de gris qui peuvent prêter à confusion. C'est pourquoi certains auteurs [73,74] préconisent l'emploi de substances qui augmentent le contraste entre les différentes structures anatomiques. Ces produits de contraste sont des substances paramagnétiques ou super paramagnétiques qui influencent l'environnement électroniques des tissus et augmentent la valeur locale du magnétisme. Les principaux produits de contraste sont des agents paramagnétiques ou super paramagéntiques.

- Les agents paramagnétiques sont des composés organiques qui se caractérisent par la présence, sur la liaison azote oxygène, d'un électron célibataire responsable de leur paramagnétisme. Exemple : les radicaux libres, le $\mathrm{Cu}++, \mathrm{Co}++$, le $\mathrm{Mn++}$. Les molécules d'eau perçoivent l'information magnétique lors de leur passage à proximité de l'électron non apparié d'où un effet sur la relaxation.

- Les agents super paramagnétiques sont des suspensions colloïdales de monoparticules de magnétite séparées par des couches perméables de dextran, le tout diffusant sous l'agitation thermique. Chacune des monoparticules super paramagnétiques agit comme un petit aimant permanent et présente donc une forte tendance à attirer et à agglomérer les autres particules.

D'un point de vue physique, les produits de contraste ont l'avantage de raccourcir le temps de relaxation longitudinal T1. Dans l'exploration de I'ATM, le produit de contraste ne trouve sa médecine buccale chirurgie buccale VOL. $8, \mathrm{~N}^{\circ}$ 2002 page 19 
médecine buccale chirurgie buccale

L. $8, \mathrm{~N}^{\circ} 1$

2002

page 20 place que dans la détection des maladies rhumatoïdes inflammatoires [73]. La méthode d'exploration mise au point par Smith ${ }^{[73]}$ consiste à pratiquer un examen avant et après injection de produit de contraste, le Gd DTPA.

L'emploi des séquences SE 600/15 et SE 200/25-90, a permis d'obtenir les résultats suivants :

- une augmentation du contraste des tissus mous entourant l'attache dorsale chez des sujets asymptomatiques ;

- une augmentation du signal de l'épanchement chez les sujets souffrant d'un dysfonctionnement ;

- une augmentation du signal de la partie ventrale du disque en état de destruction (confirmé par la chirurgie).

Les travaux de Mikhael et coll [74] montrent que le GD DTPA permet de mieux délimiter la capsule et les ligaments. L'utilisation du produit de contraste ne semble donc pas dénuée d'intérêt. Mais ce bénéfice est contrebalancé par des inconvénients majeurs. Le risque allergique, l'obligation d'une double acquisition, le coût élevé des produits de contraste doivent y faire renoncer dans la plupart des examen de l'ATM.

\section{LA PATHOLOGIE FONCTIONNELLE DE L'ATM : les dysfonctions crânio- mandibulaires, leurs conséquences anatomo-pathologiques et IRM}

Le terme de dysfonction crânio-mandibulaire (DCM) traduction de l'anglais craniomandibular disorder s'est imposé à la fin des années 80 pour désigner un ensemble de symptômes plus ou moins rapportable au dysfonctionnement de l'articulation temporo-mandibulaire. Le seul avantage de cette terminologie est de laisser ouverte la question de savoir si ces symptômes peuvent se systématiser en syndrome, ce que I'on croyait jusqu'alors (Costen, Sadam). II reste que la définition des DCM est loin d'être satisfaisante. Rappelons pour mémoire celle donnée par Hartmann [83] : «douleurs et ensemble des troubles liés au dysfonctionnement de l'articulation temporo-mandibulaire».

\section{Approche statique des dysfonctions crânio-mandibulaires}

\section{Données cliniques}

Les dysfonctions crânio-mandibulaires englobent différentes anomalies de relation entre la mandibule et la surface articulaire temporale dont l'expression s'effectue le plus souvent au cours des mouvements articulaires. Ces dysfonctions impliquent bien sûr d'emblée ou au décours de leur évolution la mise en jeu du disque articulaire.

On peut distinguer 3 types d'anomalies dysfonctionnelles de l'ATM. On les classe habituellement par ordre de gravité croissante :

- la luxation discale antérieure, réductible à l'ouverture buccale,

- la luxation discale antérieure irréductible quel que soit le degré d'ouverture buccale,

- la luxation discale antérieure s'accompagnant d'une perforation du complexe disco-ligamentaire.

Ces luxations se caractérisent par des symptômes subjectifs comme les algies, les acouphènes et des symptômes objectifs qui signent les anomalies de relation entre le disque et les éléments qui l'entourent. Ce sont ces signes objectifs cliniques tels que les hernies mandibulaires, les ressauts, les dyskinésies, les crépitations ou les claquements qui vont se concrétiser par des images de déplacement discal antérieur ou médio-latéral (interne ou externe) à l'ouverture comme à la fermeture buccale [84]. L'exploration de l'ATM par l'IRM permet, grâce à la visualisation du disque de connaître la position et l'éventuel déplacement du disque par rapport au condyle mandibulaire et au tubercule articulaire du temporal.

- Au cours de la luxation antérieure réductible sont retrouvés les bruits articulaires (claquement et craquement) associés ou non à une douleur. L'ouverture buccale dans ce cas n'est pas limitée. Le déplacement du disque vers l'avant lors de la fermeture buccale provoque un bruit perçu le plus souvent lors de la réduction de la luxation à l'ouverture, celui-ci peut survenir également à la fois à l'ouverture et à la fermeture buccale il s'agit alors du claquement 
réciproque de la récidive luxante. Ce bruit est le résultat de la friction entre le bourrelet postérieur discal et le condyle mandibulaire lorsqu'ils se déplacent dans des directions opposées et que le disque retrouve sa position normale supra condylienne. II apparaît dans la même configuration que celle d'une ATM asymptomatique BF [85].

- Au cours de la luxation antérieure non réductible le disque ne retrouve pas sa position supra condylienne à l'ouverture et demeure en avant du condyle ; ll se produit alors un étirement du frein discal dorsal responsable de douleurs car le disque forme un obstacle mécanique à la translation antérieure du condyle et limite par conséquent l'ouverture buccale. Aucun bruit articulaire n'est perçu.

- La luxation antérieure non réductible peut conduire à une perforation disco-ligamentaire et touche principalement le ligament discal postérieur [51-53]. Cette perforation peut occasionnellement intéresser le disque qui alors ne joue plus le rôle d'une butée limitant la course du condyle. Les surfaces osseuses se trouvant au contact l'une de l'autre, il s'ensuit des lésions osseuses dégénératives caractérisées par des bruits à type de crépitation.

- A côté de ces luxations discales antérieures

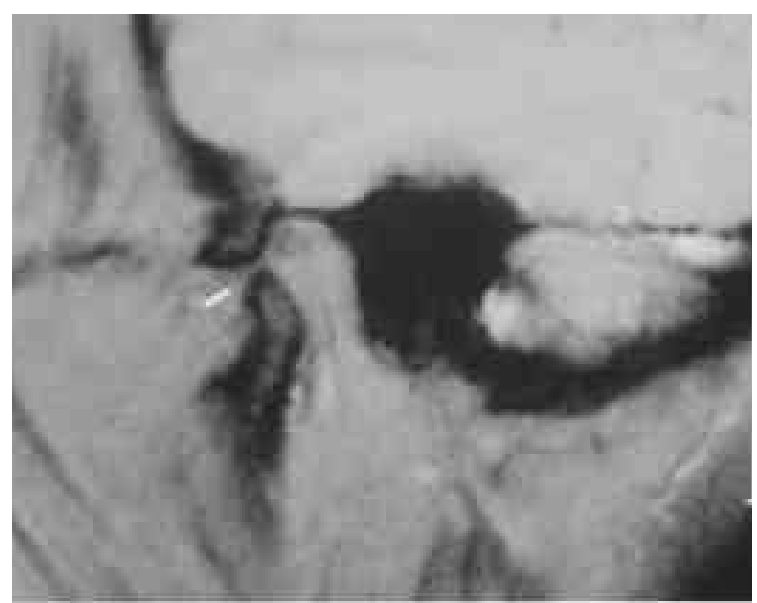

Figure 10 : Coupe sagittale bouche ouverte du patient présentant une luxation discale antérieure réductible. Noter l'hyposignal discal au dessus de la tête du condyle (flèche).

Sagittal image in a opened-mouth position reveals displaced disk with recapture. Note the discal hyposignal above the condyle head (arrow). certains auteurs ont décrit des déplacements partiels latéraux et en rotation du disque. Les travaux de Pujol [86] ont montré que sur 700 ATM dysfonctionnelles étudiées, sont reconnaissables des luxations frontales isolées, des déplacements en rotation du disque et des luxations antérieures partielles concernant la partie latérale de l'ATM.

\section{Imagerie Statique des DCM}

\section{- Images des différentes luxations}

Ces dérangements internes ont fait l'objet d'investigations selon des méthodes variées. Les travaux comparatifs entrepris par plusieurs auteurs, ont montré que l'IRM est une méthode non seulement non invasive mais de surcroît plus confortable pour le patient car une étude bi planaire peut se faire sans changement positionnel [11,12,34,35,53,87-90].

Les images fournies par l'IRM en position BO et $B F$ de ces dérangements internes définissent le disque par un hyposignal de forme lenticulaire situé entre les 2 surfaces articulaires. Cette image dite en hyposignal dans la partie discale la plus épaisse morphologiquement correspond au sommet de la fosse mandibulaire. La partie discale correspondant à la jonction disco-ptérygoïdienne apparaît identique mais moins

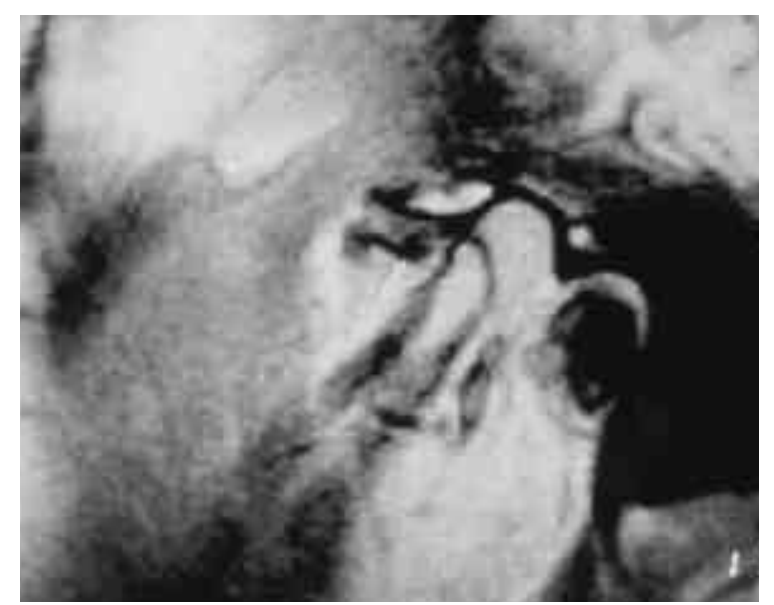

Figure 11 : Coupe sagittale bouche ouverte montrant une luxation discal avant (flèche) non réductible. Sagittal image in a opened mouth position reveals displeaced disk (arrow) without recapture. médecine buccale chirurgie buccale VOL. $8, \mathrm{~N}^{\circ}$ 2002 page 21 
médecine buccale chirurgie buccale constante. Dans un contexte pathologique la luxation discale antérieure réductible est carac térisée par un hyposignal situé au-dessus du condyle en position bouche ouverte (Fig.10) ; tandis qu'il est en avant de celui-ci dans le cas d'une luxation discale antérieure non réductible dans la même position buccale. (Fig. 11)

Certains auteurs ont décrit des changements histologiques ou/et biochimiques au sein même du disque et de la zone bilaminaire. Celle-ci est constituée d'une bande épaisse de tissu conjonctif lâche, vascularisé et bien innervé, ce qui la distingue de la partie discale proprement dite. L'exploration IRM d'ATM présentant des dérangements internes importants montre à l'évidence une absence de démarcation lisible entre ces deux éléments [6], c'est un signe objectif supplémentaire qui contribue à préciser le diagnostic.

Pour l'étude des déplacements partiels, latéraux et en rotation du disque, il est essentiel de pratiquer des coupes sagittales et coronales lors de l'exploration des ATM dysfonctionnelles par le moyen de l'IRM dans le but d'étudier les déplacements discaux dans les 3 plans de l'espace à un temps t (Fig. 12a et b). II semble que certains échecs thérapeutiques de recapture du disque articulaire soient dus à un manque de précision dans la localisation du disque [86].

Afin d'accroître la précision du positionnement discal par rapport au condyle, Gabler MT et coll [91] ont imaginé une construction géométrique. Ils ont utilisé pour cela un système de coordonnées $X Y$ où le tubercule articulaire et la fosse mandibulaire sont des références stables. La figure 13 montre que le point $00^{\prime}$ représente le point le plus profond de la fosse mandibulaire à partir duquel tous les mouvements du complexe condylo-discal sont mesurés à la fois dans les positions BO et BF. Le point OX est le point le plus inférieur de la surface articulaire du tubercule articulaire, la ligne perpendiculaire à I'axe OO'- OX étant OY. Après exploration de I'ATM par I'IRM, l'auteur a comparé les images du disque en position BO et BF par rapport à ces axes avant et après chirurgie arthroscopique. L'analyse de ces images a pu mettre en évidence trois types de relations entre les trois éléments anatomiques que sont la fosse mandibulaire, le condyle et le disque :

- une relation entre le condyle et la fosse temporale,

- une relation entre le disque et la fosse temporale,

- une relation entre le disque et le condyle.

Ce montage géométrique n'a cessé depuis de voir son champ d'application s'élargir.

\section{- Applications à la thérapeutique}

Du point de vue thérapeutique, la situation discale par rapport à la fosse mandibulaire et au condyle décrite précédemment, peut être utile pour la confection et la mise en place de gouttières occlusales ${ }^{[92]}$ celles-ci n'étant qu'un moyen d'adapter entre elles les structures articulaires environnantes lors des dérangements internes (Fig. 14). II faut préciser cependant, que la gouttière occlusale ne permet pas une réduction de la luxation chez les sujets souffrant depuis longtemps d'un dysfonctionnement [93]. Pour les cas dont la pathologie est plus récente, I'IRM n'est qu'un moyen de contrôle et de surveillance post-thérapeutique complémentaire [94-97].

D'autre part l'IRM a permis d'avancer les premières hypothèses concernant les effets des thérapeutiques non chirurgicales et d'expliquer en partie leur efficacité. Choi et coll [98] ont pratiqué des examens en IRM avant et après traitement non chirurgical d'une luxation. Ils ont pu montrer que le ménisque ne reprend pas réellement sa position physiologique, mais que le rétablissement est le fait d'une plus grande mobilité du condyle ainsi que d'une adaptation de l'attache postérieure.

\section{- Evaluation post orthodontique des ATM}

La pathologie articulaire et ses conséquences cinesthésiques ont intéressé les orthodontistes qui ont cherché à déterminer la prévalence des dérangements internes dans la population juvénile avant traitement orthodontique. Les travaux

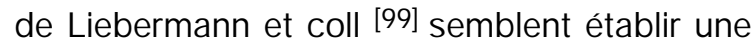
corrélation entre les résultats de I'IRM et les symptômes douloureux neuromusculaires lors de la palpation. L'exploration des ATM chez les sujets jeunes avant la planification d'un traitement orthodontique semble donc importante puisque des luxations asymptomatiques sans 

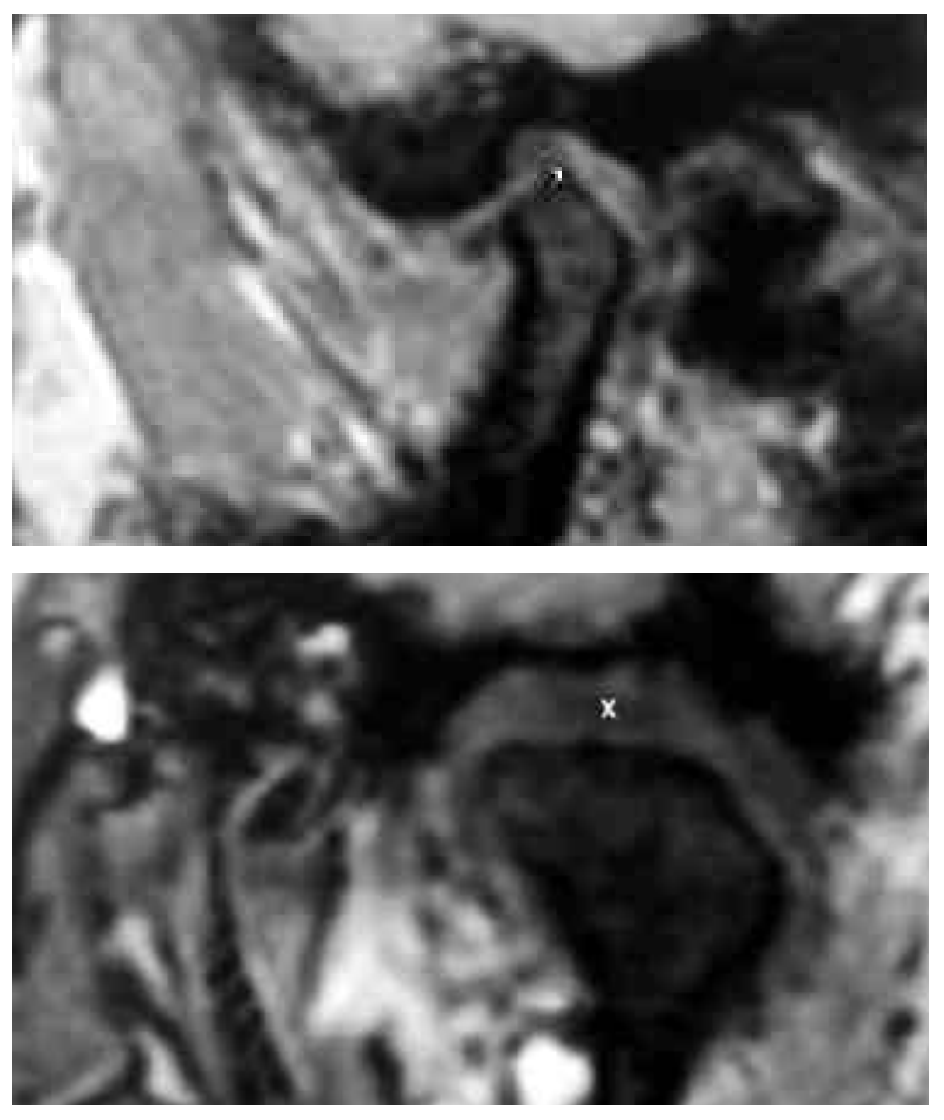

Figure 12b : Coupe coronale de l'ATM. Noter la présence de l'hyposignal du disque (croix)

Coronal image of the TMJ. Note the presence of the discal hyposignal (cross).
Figure 12a : Coupe sagittale d'une ATM asymptomatique
Sagittal image of asymptomatic TMJ

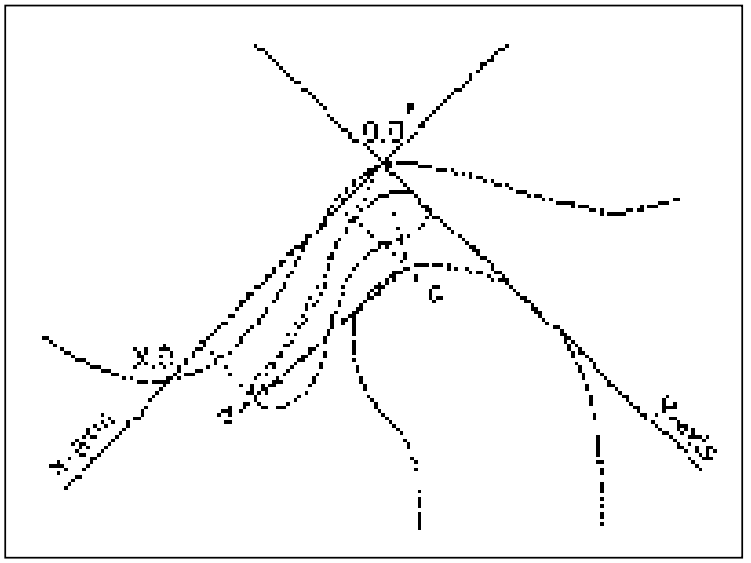

Figure 13 : Repère orthogonal $X, O, O^{\prime}, Y$ avec :

$C$ : point de référence condylien

$D$ : point de référence méniscal

$0,0^{\prime}$ : point le plus profond de la cavité glénoïde (Gabler 1989)

$\mathrm{X}, \mathrm{O}, \mathrm{O}^{\prime}, \mathrm{Y}$ coordinate system designed by using the articular eminence and the glenoïd fossa has stabled reference landmarks, with :

C : condyle referentiel point

$D$ : articular disk reference point

$0, \mathrm{O}^{\prime}$ : the midpoint of the most superior glenoïd fossa depth (Gabler 1989)

\section{médecine buccale chirurgie buccale \\ VOL. $8, \mathrm{~N}^{\circ}$ 2002}

page 23

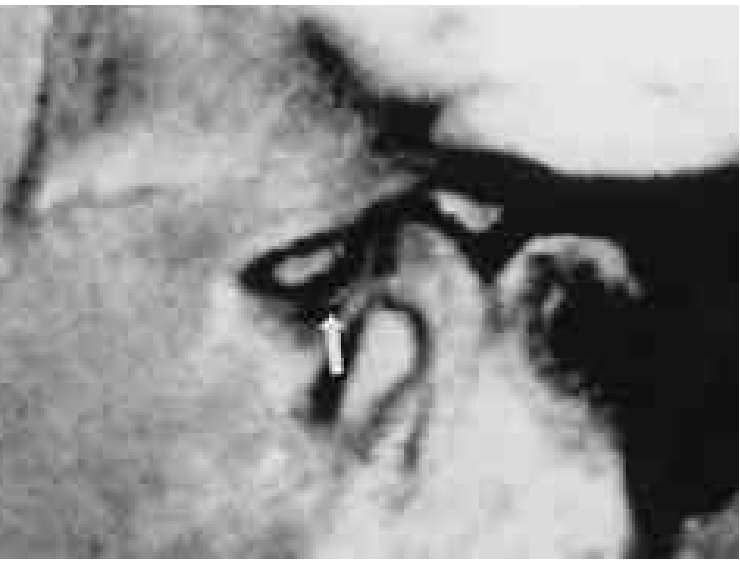

Figure 14 : Repositionnement du disque (flèche) grâce à une gouttière chez un patient présentant une luxation discale réductible à l'ouverture.

Disck repositioning (arrow) with splint cast treatment. 


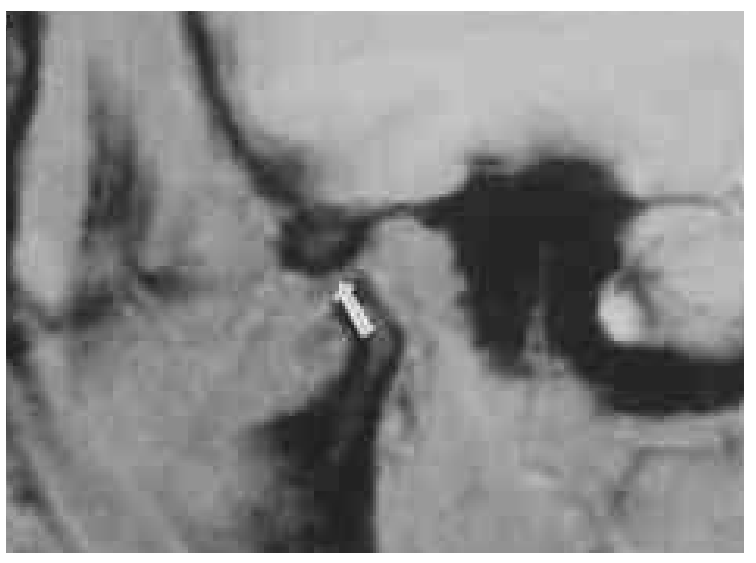

Figure 15a : Bouche fermée : I'hyposignal du disque (flèche) est en avant de la tête condylienne.

Closed mouth position : discal hyposignal is in front the condyle head (arrow).

médecine buccale chirurgie buccale

L. $8, \mathrm{~N}^{\circ} 1$

2002

page 24

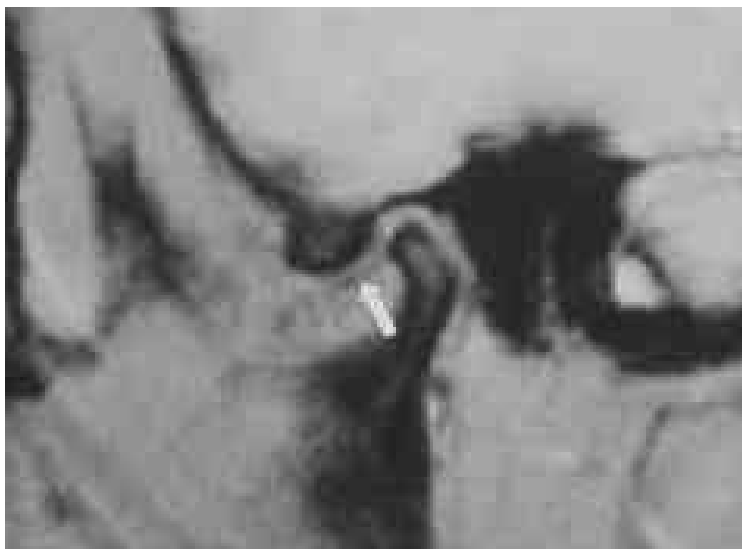

Figure 15b : Bouche ouverte à $25 \mathrm{~mm}$ : I'hyposignal du disque est au dessus de la tête condylienne (flèche).

$25 \mathrm{~mm}$ opened mouth position : discal hyposignal is above the condyle head (arrow).

réduction peuvent engendrer des douleurs dans la phase active du traitement orthodontique. Kircos et coll [100] ont d'ailleurs établi qu'une position antérieure du disque chez les sujets asymptomatiques serait un facteur prédisposant au dysfonctionnement de l'ATM.

\section{Approche cinétique des DCM}

L'étude dynamique en IRM permet de suivre le déplacement du disque au cours du cycle d'ouverture-fermeture buccale. II est ainsi aisé, dans

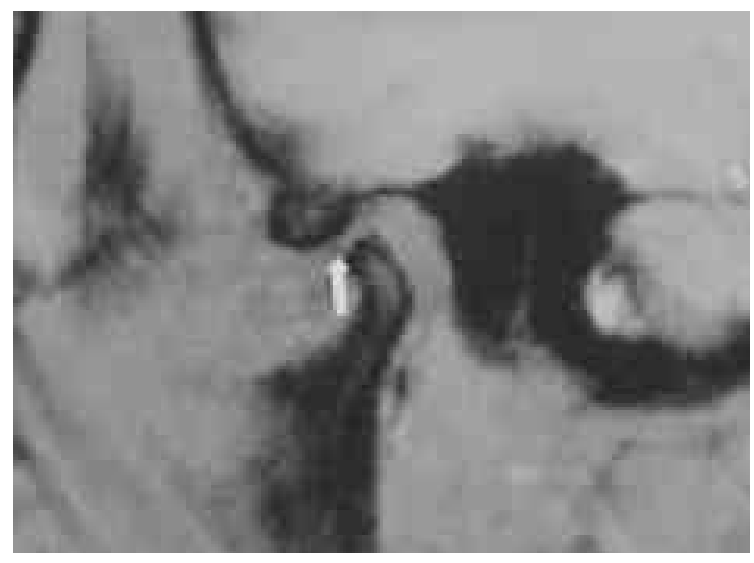

Figure 15c : Bouche ouverte à $18 \mathrm{~mm}$ : début du ressaut condylien.

$18 \mathrm{~mm}$ opened mouth position: the start of the jaw clicking.

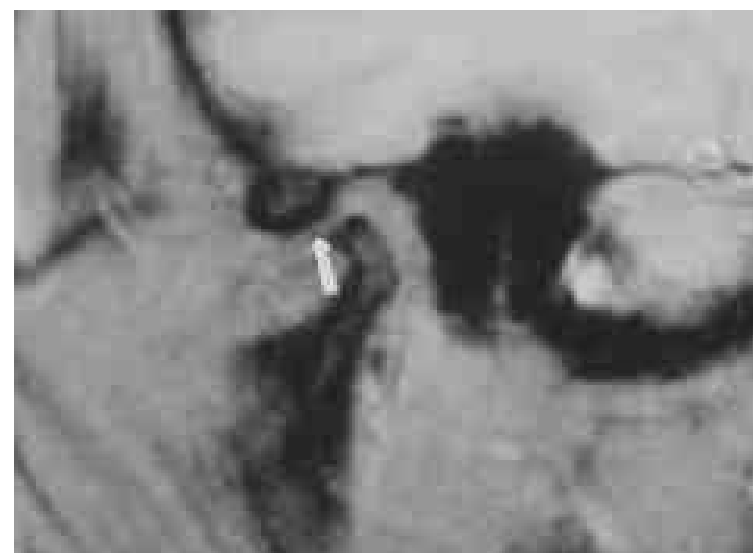

Figure 15d : Début de la fermeture buccale. Le condyle se retrouve après fermeture totale de la bouche en arrière du disque (voir flèche).

The begining of the closing mouth. The condyle is completely behind the disk (arrow).

les situations dysfonctionnelles, de déterminer le moment exact où le disque quitte sa position supra condylienne pour se déplacer vers l'avant lors de la fermeture buccale (Fig. 15a,b,c,d). L'IRM permet de repérer le moment exact de la recapture du disque. Les thérapeutes ont ainsi le choix de s'orienter soit vers un traitement conservateur soit vers une solution chirurgicale. Dans un cas comme dans l'autre l'IRM semble s'imposer comme moyen indispensable dans le diagnostic différentiel des luxations ${ }^{[101,102]}$ ainsi que dans la 


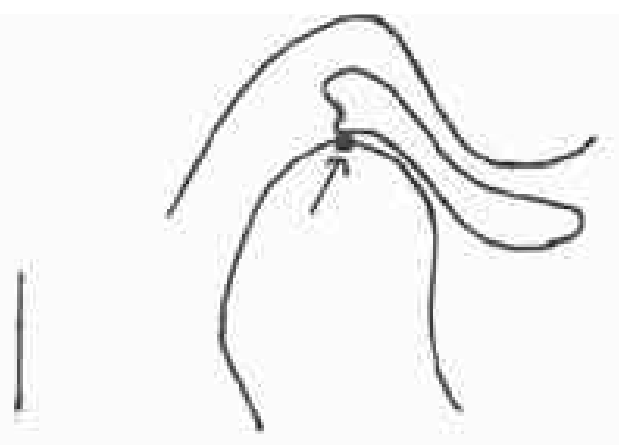

a

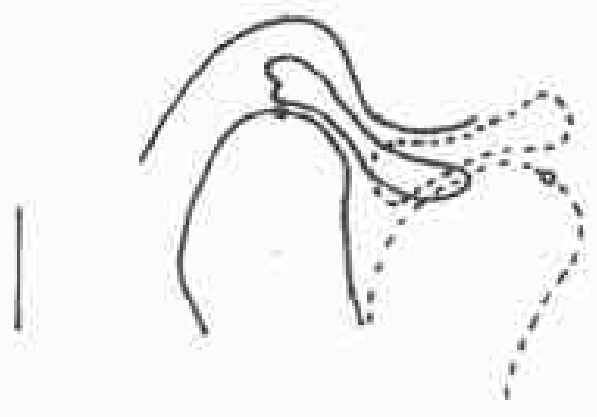

C

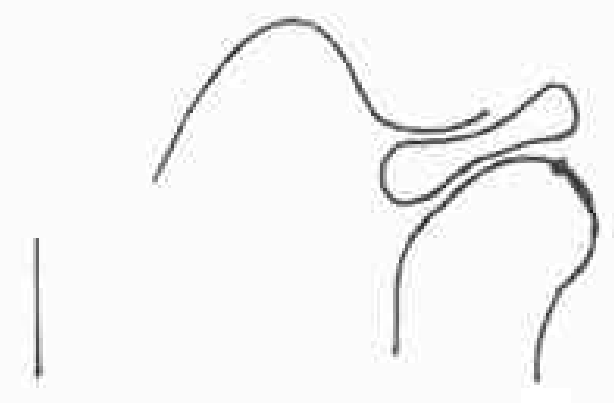

b

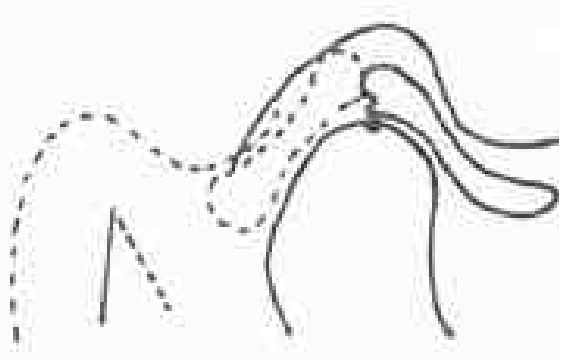

d

Figure 16 : Schéma montrant la relation entre le condyle mandibulaire le ménisque et le condyle temporal :

a) tracé en intercuspidation maximale

b) tracé en occlusion centrée

c) tracé en pointillé du complexe condylo-méniscal dans la position BO. Enregistrement par rapport au condyle temporal

d) tracé en pointillé du complexe condylo-méniscal dans la position BO. Enregistrement par rapport à la mandibule

Relationship between the mandibular condyle the disk and the eminence :

a) draw in maximun intercuspation

b) draw in centric occlusion

c) dotted draw of the condylar disk system in opened mouth position. Recorded in relation to the eminence

d) dotted draw of the condylar disk system in opened mouth position. Recorded in relation to the mandibule

planification des interventions chirurgicales [103]. En effet, l'animation des images d'une série de coupes acquises à différentes phases du cycle articulaire, permet de visualiser les différents types de luxations et de préciser l'instant où elles surviennent. Les séquences rapides en EG ont permis de limiter le temps d'examen [20,31,104107], tout en quantifiant le mouvement du disque par rapport aux composants osseux.

Price [108] a mis au point une méthode originale : les images issues de l'exploration de I'ATM sont agrandies; un point de référence est pris au sommet du condyle en position BF ; le tracé des trois éléments qui constituent l'ATM (l'os temporal, le disque et le condyle) pris dans cette position BF est superposé aux mêmes éléments dans la position BO. La translation du condyle est indiquée par la distance entre les deux positions du point de référence et par l'angle de rotation égal à celui de la mandibule. Ainsi la position du disque peut être précisée par rapport aux deux surfaces articulaires. Un facteur d'agrandissement permet la corrélation de ces mesures (Fig. 16). médecine buccale chirurgie buccale VOL. $8, \mathrm{~N}^{\circ}$ 2002 page 25 
médecine buccale chirurgie buccale

L. $8, \mathrm{~N}^{\circ} 1$

2002

page 26
Néanmoins, le tracé sur image des différents éléments de l'ATM et la précision des mesures requises sont des sources d'erreur qui impliquent l'utilisation de moyens informatiques. Ainsi, le logiciel de mode pseudo cinématique (Siemens ${ }^{\circledR}$ ) permet l'enregistrement phase par phase du cycle articulaire comme le définit Rees [109] dans le plan sagittal. La reproduction d'un cycle d'ouverture-fermeture avec une vitesse de défilement réduite des images, contribue à bien visualiser chaque phase tout en conservant au déroulement du cycle son caractère animé. Lors d'une étude dynamique d'une ATM dysfonctionnelle, seules certaines phases du mouvement sont à privilégier, en particulier la phase où survient la luxation ; l'intégralité du mouvement n'étant pas nécessaire au diagnostic.

Cependant une objection fondamentale doit être faite à cet enregistrement phase par phase. Elle est liée à la technique elle-même, pseudo cinématique et ne peut donc reproduire un mouvement continu, par conséquent, physiologique.

L'amélioration des techniques IRM et des logiciels informatiques a permis à Pujol [86] de procéder à l'étude dynamique des ATM en séquence Fast Spin Echo et en densité protonique. Un cycle d'ouverture-fermeture buccale a pu être exploré en temps réel. L'auteur souligne l'intérêt de l'étude dynamique en temps réel dans la recherche du timing de l'antéposition discale dans le cas des luxations réductibles. Cette méthode peut être étendue à l'espace tridimensionnel, ce qui suppose des examens en mode 3 DFT plus longs. Bien que ces méthodes d'analyses semblent apporter des éléments nouveaux dans la connaissance du trajet discal, lors du mouvement articulaire, certains auteurs restent sceptiques quant à l'efficacité de l'IRM dans la visualisation des différents éléments anatomiques de l'ATM $[7,110]$.

\section{IRM ET TRAUMATOLOGIE DE L'ATM}

\section{Les traumatismes extrinsèques}

Dans certaines manipulations d'ordre thérapeutique telles que les extractions de la $3^{\mathrm{e}}$ molaire, le traitement orthodontique crânio-facial [111], on peut être confronté à de sévères désordres articulaires. Ces dysfonctionnements iatrogènes ne sont cependant pas courants. En revanche, dans les accidents de la route, le «coup du lapin» est un traumatisme fréquent, sa visualisation serait problématique sans l'aide de l'IRM. Pressman et coll [112] grâce à différentes images obtenues dans un plan transversal, sur des coupes de $2 \mathrm{~mm}$ et avec des séquences à TR et TE longs ou courts, ont décrit, en 1992, les aspects biomécaniques de ce traumatisme appelé «whiplash» par les anglo-saxons. Les auteurs ont pu mettre en évidence et décrire la localisation du disque, la zone d'épanchement fluide, et la fracture intracapsulaire du condyle. Celle-ci peut engendrer à la suite du choc, une compression des tissus rétro discaux entre la tête condylienne et le plateau tympanique ce qui a pour résultat la rupture de l'attache postérieure du disque.

Les fractures condyliennes de tout type sont évidemment mieux explorées en TDM qu'en IRM, Buthiau et coll [113] relèvent néanmoins la possibilité de diagnostiquer en IRM les lésions éventuelles de l'appareil disco-ligamentaire associées à la fracture. Cet auteur cite notamment le cas des hémarthroses associées à une fracture intra capsulaire du condyle mandibulaire : en IRM les épanchements hématiques sont hypo-intenses en $\mathrm{T} 1$ et $\mathrm{T} 2$ lorsqu'ils sont récents, puis deviennent progressivement hyper intenses au cours du processus de dégradation de l'hémoglobine. L'intérêt d'un tel examen est de poser l'indication de drainage chirurgical de l'hémarthrose.

König et coll $[69,70]$, ont utilisé les déplacements chimiques en IRM pour évaluer les anomalies de I'ATM. Dans les conditions pathologiques extrêmes, caractérisées par la présence de fluide inflammatoire, les séquences SE à TR et TE longs sont les plus indiquées [71,72]. Les signaux qui en résultent, en particulier dans les différentes effusions de l'ATM, sont définis par rapport au signal de la graisse (grise) [57-62]. L'ankylose est une pathologie principalement d'origine traumatique de l'articulation temporomandibulaire. Cependant elle peut être égale- 
ment la conséquence fréquente d'une arthrite infectieuse non traitée ou résulter d'étiologies plus rares telles les arthropathies systémiques ou les ostéo-chondromes [114]. Le symptôme dominant est la limitation lentement progressive et à peine ou pas du tout douloureuse de la mobilité articulaire qui finit par se transformer en un blocage articulaire total. Au contraire de ce qui se produit dans les limitations d'ouverture d'origine non articulaire (par exemple de cause musculaire) la mobilité est réduite dans toutes les directions de l'espace : les mouvements de latéralité et de protrusion deviennent limités puis impossibles.

Sur le plan histo-pathologique, l'ankylose se traduit par la réduction progressive des interlignes articulaires qui disparaissent totalement dans l'ankylose osseuse ou qui persistent de façon vestigiale dans les ankyloses conjonctives. Rowe [115] a isolé au sein des ankyloses conjonctives les ankyloses fibreuses, fibroosseuses, et cartilagineuses.

Parmi les traumatismes causaux, on relève bien sûr les accidents du trafic mais aussi et de façon non négligeable les fractures obstétricales et les fractures du jeune âge passées totalement inaperçues. Enfin, la chirurgie de I'ATM en particulier les condyloplasties, et la chirurgie orthognathique lorsqu'elle est accom-

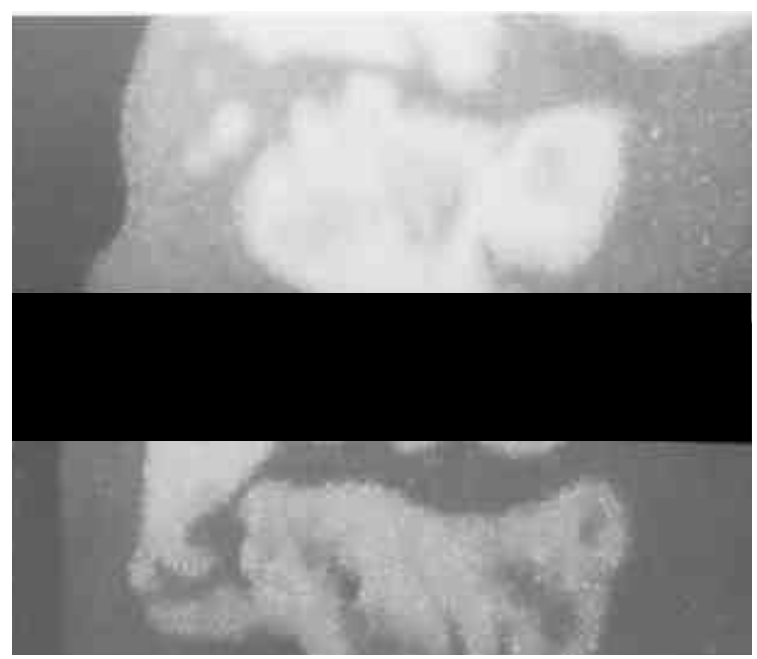

Figure 17 : Coupe coronale en TDM d'une ATM présentant une arthrose dégénérative post-traumatique. Post traumatic degenerated arthrosis of TMJ detected by computed tomography. Coronal section. pagnée d'un blocage intermaxillaire prolongé sont l'origine d'un nombre significatif de cas d'ankylose de l'ATM (Fig. 17).

Sur le plan de l'imagerie, la tomodensitométrie est la plus indiquée en cas de suspicion d'ankylose osseuse, puisque le compartiment fibrocartilagineux a complètement disparu. Par contre dans les ankyloses conjonctives, l'IRM a fait la preuve de sa supériorité [116]. En cas d'ankylose fibreuse, I'IRM montre une transformation fibreuse de l'espace condylo-temporal, une adhérence discale sur le versant temporal de l'articulation, et confirme l'absence de translation du condyle mandibulaire [113].

\section{Les microtraumatismes intrinsèques}

La traumatologie induite par nos différentes manipulations ou d'origine accidentelle, ne doit pas occulter les traumas dus spécifiquement au dysfonctionnement de l'articulation à savoir les perforations et les adhérences discales.

L'IRM apporte-t-elle une contribution dans la détection de cette pathologie?

Précisons d'emblée, que concernant les perforations, les avis divergent. Pour Rao [95] ce qui apparaît comme perforation discale à l'IRM n'est en fait que l'image très atténuée de la zone bi laminaire étirée dans les déplacements discaux. En revanche, Nokes et coll [117] après une étude faite sur vingt patients, constatent que l'image discontinue du disque observée en IRM ainsi que l'érosion du tubercule articulaire et la perte d'espace articulaire signent dans $86 \%$ des cas une perforation discale.

Les adhérences discales qui se traduisent par la fixité du disque dans sa relation avec la fosse mandibulaire et le tubercule articulaire dans les deux positions $B O$ et $B F$, sont mises en évidence par le truchement d'une construction géométrique. Elles sont par conséquent révélées indirectement par I'IRM. C'est Rao et coll [94] qui ont mis au point la méthode. Elle consiste à tracer sur l'image une ligne verticale qui passe par la bande postérieure jusqu'à l'intersection avec le contour de la fosse mandibulaire, une autre ligne part de la bande antérieure et se termine juste au niveau du tubercule articulaire. Ces tracés se font dans les positions médecine buccale chirurgie buccale VOL. $8, \mathrm{~N}^{\circ}$ 2002 page 27 
$\mathrm{BO}$ et BF. Le moindre changement de position du disque par rapport à ces repères représente une mobilité. Dans le cas contraire, le disque est considéré comme fixe. Cette analyse rationnelle conduit à une nouvelle approche dans I'interprétation des images en IRM. Elle permet de distinguer six cas différents concernant la position du disque dans les dérangements internes de l'ATM :

- le disque est en position normale et mobile,

- le disque est en position normale mais adhérente,

- le disque est déplacé mais mobile avec réduction,

- le disque est déplacé mais mobile sans réduction,

- le disque est déplacé mais adhérent avec réduction,

- le disque est déplacé mais adhérent sans réduction.

médecine buccale chirurgie buccale

L. $8, \mathrm{~N}^{\circ} 1$

2002

page 28

\section{LA PATHOLOGIE INFECTIEUSE DE L'ATM}

L'articulation temporo-mandibulaire peut être le siège de processus infectieux par contamination locale régionale ou systémique. Les infections par contigüité ont pour origine les infections de la région parotidienne (parotidite) de l'oreille moyenne (otite moyenne), les infections odontogènes (ostéomyélites mandibulaires). Les otites moyennes de l'enfance sont une cause fréquente et souvent inaperçue d'arthrites septiques de l'ATM qui aboutissent à une atteinte du centre de croissance condylien qui peut se manifester par un ralentissement unilatéral de la croissance mandibulaire. Les infections systémiques, à staphylocoques ou à streptocoques pour l'essentiel, ont fortement régressé avec l'avènement des antibiotiques. La traumatologie mandibulaire avec fracture du col du condyle déplacée et «embarrure» du méat acoustique externe peut s'accompagner d'une contamination aiguë par effraction de la capsule.

Dans le cas d'atteintes infectieuses, I'IRM révèle des perturbations tissulaires qui se traduisent au niveau de l'os par un état dégénératif qui peut aboutir à la formation marginale d'ostéophytes, à une sclérose ou à la formation de kystes subcorticaux. Au niveau du disque, on assiste à une altération des fibres collagènes qui se traduit par un hyposignal pour des séquences pondérées T1 ou par un signal variable pour des séquences pondérées $\mathrm{T} 2$.

\section{LA PATHOLOGIE INFLAMMATOIRE ET DÉGÉNÉRATIVE}

\section{Pathologie inflammatoire rhumatoïde}

La pathologie inflammatoire rhumatismale a un retentissement au niveau de I'ATM comme au niveau de la plupart des articulations. L'arthrite rhumatoïde, la spondylarthrite ankylosante, l'arthrite psoriasique ou le lupus érythémateux se manifestent ainsi au niveau de I'ATM. Malheureusement, le diagnostic différentiel IRM de ces différentes maladies est souvent malaisé car leurs sémiologies IRM sont proches.

L'arthrite ou polyarthrite rhumatoïde est une polyarthrite chronique bilatérale symétrique caractérisée par une synovite proliférative hypertrophique. Les patients présentent des érosions radio visibles des surfaces articulaires et ont un test de Waler-Rose positif (facteur rhumatoïde) (Fig. 18). La synoviale est infiltrée par des nodules riches en cellules lympho-plasmocytaires. L'atteinte de l'ATM concerne 50 à $60 \%$ des patients atteints d'arthrite rhumatoïde [118-119]. La symptomatologie est dominée par la douleur et la limitation progressive de l'ouverture qui s'accompagnent d'une érosion rapide des surfaces articulaires et de l'os sous jacent avec destruction progressive du disque (Fig. 19).

L'IRM montre une irrégularité des surfaces articulaires et un pincement de l'interligne articulaire avec antéposition discale. La perte du signal normal intra-spongieux est limitée. La déformation du condyle mandibulaire est fréquente. Le pannus synovial caractéristique de la polyarthrite rhumatoïde est souvent mis en évidence (Fig. 20).

La spondylarthrite ankylosante est une entité bien à part des précédentes, puisqu'elle constitue avec l'arthrite psoriasique, le syndrome de Fiessinger-Leroy-Reiter et avec les arthrites associées aux pathologies inflammatoires intes- 


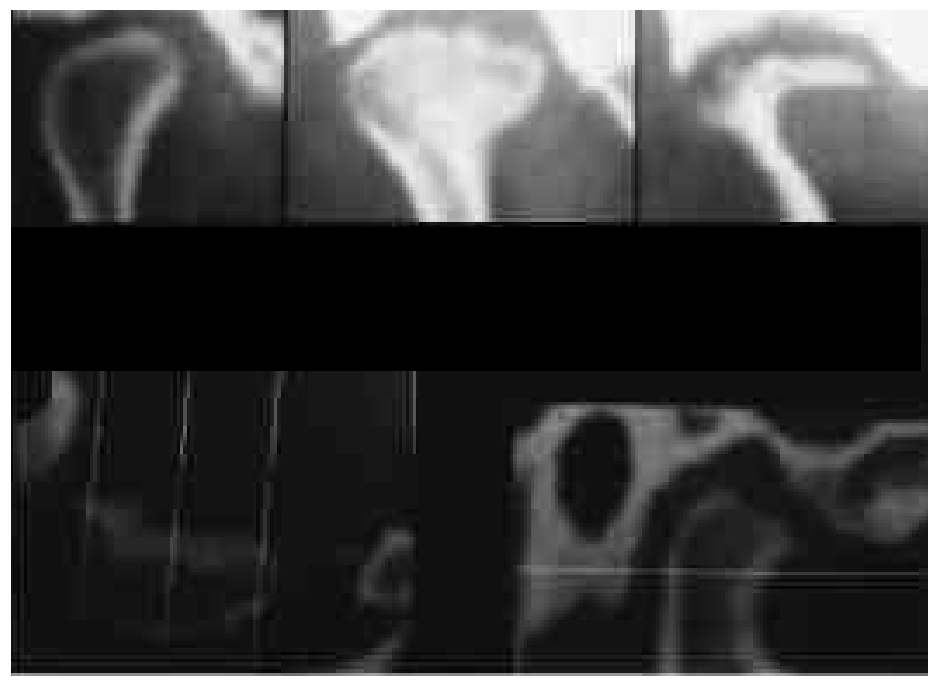

Figure 18 : Coupes coronale et sagittale en TDM d'une ATM présentant une arthrite rhumatoïde.

Rheumatoïd arthritis of TMJ detected by computed tomography. Sagittal and coronal sections

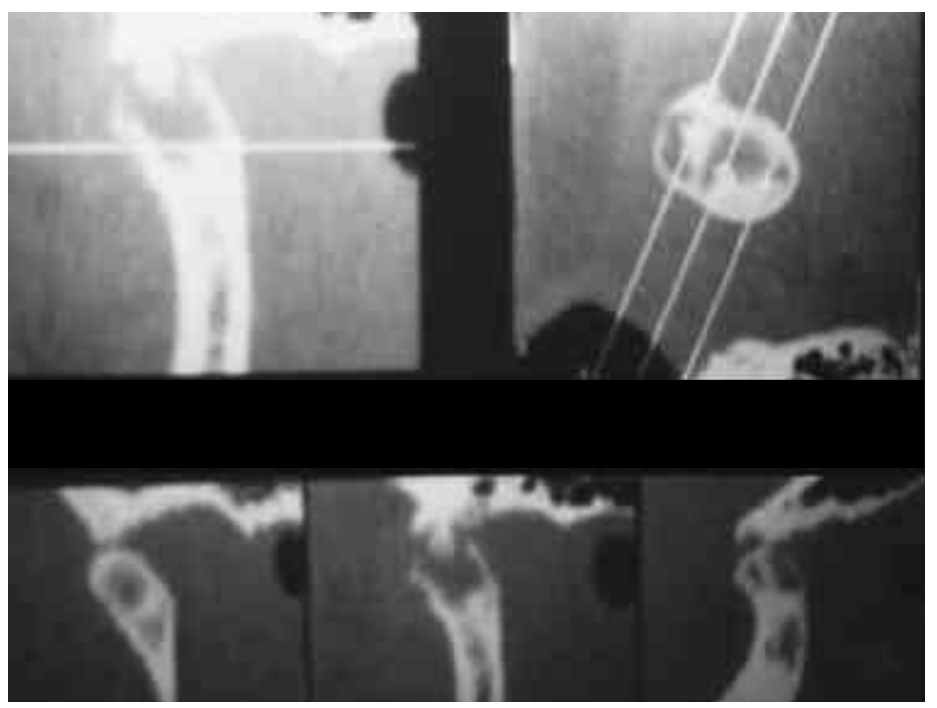

Figure 19 : Coupes sagittales en TDM d'une ATM présentant une polyarthrite rhumatoïde.

Rheumatoïd polyarthritis of TMJ detected by computed tomography. Sagittal sections.

médecine buccale chirurgie buccale

VOL. $8, \mathrm{~N}^{\circ}$ 2002

page 29

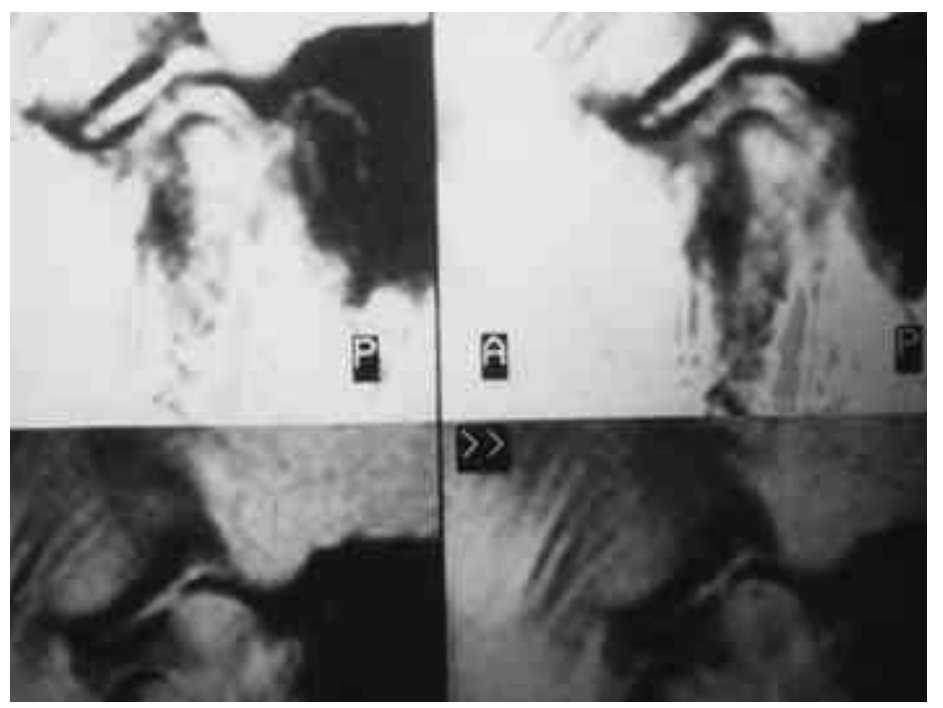

Figure 20 : Séquence dynamique en IRM d'une ATM présentant une polyarthrite. Dynamic sequence of an affected TMJ by rheumatoïd polyarthritis detected by computered tomography. 
médecine buccale chirurgie buccale

)L. $8, N^{\circ} 1$

2002

page 30 tinales un ensemble d'arthrites séronégatives lors de la recherche du facteur rhumatoïde. La spondylarthrite ankylosante est une pathologie inflammatoire chronique des articulations vertébrales et sacro-iliaques au cours desquelles la douleur et la rigidité nocturne s'associent à une incurvation progressive et à une limitation de la mobilité de l'axe vertébral. Les patients présentent le gène HLA B27 sur le $6^{\text {e }}$ chromosome. Les articulations non vertébrales comme l'ATM peuvent être atteintes.

L'IRM montre l'irrégularité des surfaces articulaires, la réduction de la course condylienne alors que le disque est souvent en situation normale. L'IRM retrouve les destructions de la corticale antérieure de la tête mandibulaire, la limitation de la course condylienne. Les signes dégénératifs s'étendent à la médullaire condylienne et s'accompagnent d'un épaississement cortical rapidement irrégulier.

L'arthrite psoriasique est l'association d'un psoriasis cutané ou unguéal avec une arthrite rhumatoïde. L'arthrite survient généralement après la dermatose. Elle concerne $6 \%$ des patients atteints de psoriasis. L'atteinte des articulations inter phalangiennes distales est un signe très évocateur. Au niveau de l'ATM, Stimson et Leban [120] ont décrit une ankylose récidivante chez un patient atteint de rhumatisme psoriasique. Celleci s'accompagne souvent de remodelages précoces asymptomatiques. La tomodensitométrie s'est révélée particulièrement efficace dans la mise en évidence de ces changements précoces. Les épanchements intra-articulaires classiques dans cette pathologie sont mis en évidence en T2. On note enfin des signes de constriction [121].

\section{Pathologie dégénérative}

L'Arthrose est une pathologie dégénérative du cartilage articulaire que l'on peut rencontrer improprement sous le terme d'ostéoarthrite empruntée aux anglo-saxons. Elle est caractérisée par une destruction initiale et primitive des cartilages articulaires, une ostéophytose marginale, une ostéosclérose sous chondrale avec présence de cavités pseudo-kystiques. La synoviale est en général normale ou légèrement inflammatoire.
L'arthrose de l'ATM touche principalement les femmes. Les douleurs se manifestent au repos et lors de la mobilisation qui s'accompagne d'une crépitation en fin de déplacement. Le mouvement est d'ailleurs limité. L'ouverture buccale est réduite. Les otalgies associées sont fréquentes.

La présence d'ostéophytes est un élément clé du diagnostic qui permet de séparer l'arthrose de la pathologie arthritique rhumatoïde [122,123]. A I'IRM, on observe la limitation de la course condylienne, la modification de la pente du tubercule articulaire du temporal érodé, et surtout une ostéophytose exubérante des versants ventral et dorsal du tubercule articulaire et de la tête condylienne. Les deux surfaces articulaires présentent des condensations sous-chondrales.

L'ostéochondrite disséquante ou ostéonécrose aseptique disséquante, habituellement réservée au genou de l'adolescent de sexe masculin, peut également frapper l'ATM. Cette affection est caractérisée par la dissection d'un fragment osseux sous-chondral sous l'effet d'une nécrose osseuse aseptique. Elle est probablement liée à une ischémie post traumatique locale. Au niveau de I'ATM, cette pathologie pourrait faire suite à l'application de forces extra orales excessives (Fig. 21). A l'IRM, on retrouve une image caractéristique en cupule décapitant le condyle mandibulaire atteint.

Enfin, on a rapporté des dépôts minéraux au niveau de I'ATM : dépôts calcique, dépôts $\mathrm{d}$ 'acide urique qui conduisent à la formation de tophus autour de l'articulation. Le signal engendré par ces dépôts est faible dans les séquences pondérées $\mathrm{T} 1$ et $\mathrm{T} 2$.

\section{Atteintes Inflammatoires des muscles mobilisateurs de I'ATM}

A ces manifestations osseuses et conjonctives, il faut ajouter les atteintes du tissu musculaire bien documentées par l'IRM. Ainsi, les tendinites musculaires, les remodelages musculaires consécutifs au remodelage osseux, les spasmes musculaires causés par l'atteinte de la racine du nerf facial [124] sont autant de pathologies illustrées par l'IRM. 


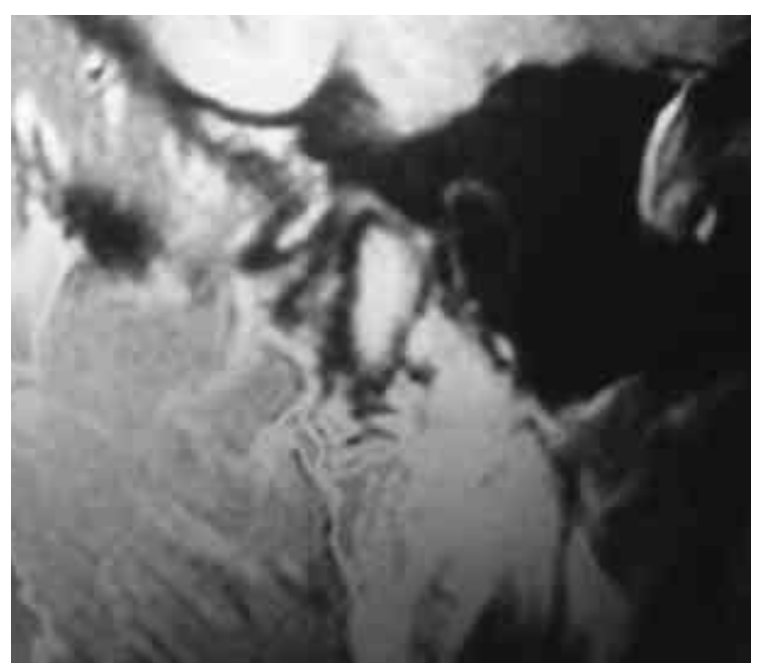

Figure 21 : Coupe sagittale en TDM d'une ATM présentant une ostéochondrite.

Ostéochondritis of TMJ detected by computed tomography. Sagittal section.

L'augmentation de fluide dans le tissu musculaire en état d'inflammation aiguë ou chronique engendrant un trismus, se traduit par une intensité de signal augmentée en séquences $S . E$ à TR et TE longs. Ceci est particulièrement vérifiable au niveau des muscles ptérygoïdiens [125]. Fulmer [43] a montré qu'une hyper laxité ligamentaire associée à une pente condylienne marquée est à l'origine d'une hyper mobilité disco-condylienne entraînant une distorsion du muscle temporal dans sa partie postérieure.

\section{LA PATHOLOGIE MALFORMATIVE}

Une anomalie dans le déroulement de l'embryogenèse ou au cours du développement peut se traduire par une pathologie malformative.

Laskin [126] a décrit les agénésies condyliennes. Elles s'accompagnent le plus souvent de déficiences des tissus mous y compris des muscles manducateurs. L'IRM est dans ce cas la principale méthode d'exploration. Elle permet d'évaluer la relation entre l'asymétrie mandibulaire d'origine congénitale et les troubles des ATM [127].

En dehors de la pathologie congénitale, la pathologie du développement s'exprime selon deux modalités : croissance excessive et croissance déficitaire.
- Des hypocycloïdes étudiées à la fois au moyen de la TDM et de I'IRM ont été rapportées dans la littérature. Cette pathologie est mise en évidence à l'adolescence mais peut également continuer à évoluer tardivement au cours de la $3^{e}$ ou de la $4^{\text {e }}$ décennie. Elle est rare et de cause inconnue. L'IRM permet de confirmer le diagnostic différentiel avec les hypertrophies condyliennes d'origine tumorale. Elle permet en particulier la mise en évidence d'amas graisseux intra-osseux qui soufflent la morphologie condylienne et constituent de véritables apophyso-mégalies [113]. La tomoscintigraphie reste néanmoins l'examen de choix pour surveiller l'évolution de l'hyperplasie condylienne.

Les asymétries musculaires d'origine congénitale comme l'hypertrophie unilatérale des muscles masticateurs ont pu être visualisées grâce à l'IRM [128]. Elles ont le plus souvent comme conséquence un modelage osseux à l'origine de la pathologie fonctionnelle.

- Les hypoplasies condyliennes non acquises sont rarement anato miquement isolées : la plupart entrent dans le cadre des microsomies crânio-faciales [129] qui regroupent l'ensemble des syndromes du premier et du second arc, les dysostoses oto-mandibulaires, les dysplasies oto-auriculo-vertébrales. Dans $70 \%$ des cas, les lésions sont unilatérales. L'hypoplasie concerne principalement la branche mandibulaire même si elle retentit sur la morphologie de toute l'hémimandibule. L'oreille se trouve abaissée et déformée, le menton et l'orifice buccal sont déplacés du côté atteint. Sur le plan osseux tous les degrés d'atteinte peuvent être rencontrés depuis une discrète asymétrie dimensionnelle du condyle jusqu'à une agénésie complète de la branche et d'une partie du corps mandibulaire. L'angle goniaque est aplati, la musculature élévatrice ipsi latérale est hypotrophique et le plan d'occlusion se trouve basculé du côté sain. Aux anomalies condyliennes et mandibulaires s'ajoutent les anomalies de l'oreille externe qui vont de l'aplasie totale du pavillon à la présence de restes embryonnaires du pavillon sur le trajet allant du tragus à la commissure labiale ipsi médecine buccale chirurgie buccale VOL. $8, \mathrm{~N}^{\circ}$ 2002 page 31 
Tableau 1 : Les différents modes d'exploration de l'ATM et leur indication.

Different modes of exploration of TMJ and their indications

médecine

buccale

chirurgie

buccale

L. $8, N^{\circ} 1$

2002

page 32

\begin{tabular}{|c|c|c|c|c|c|c|c|}
\hline $\begin{array}{l}\text { Méthodes } \\
\text { Anomalies }\end{array}$ & IRM & Arthrographie & TDM & Scintigraphie & Chirurgie & Radiographie & Tomographie \\
\hline $\begin{array}{l}\text { Luxations non réduites } \\
\text { Démarcations entre }\end{array}$ & +++ & ++ & ++ & - & +++ & + & - \\
\hline Différentes parties du disque & +++ & - & - & - & + & - & - \\
\hline Luxations réduites & +++ & ++ & ++ & - & +++ & - & - \\
\hline $\begin{array}{l}\text { Anomalies osseuses } \\
\text { de l'ATM }\end{array}$ & ++ & +++ & ++++ & +++ & +++ & + & ++ \\
\hline Aspect de la bande antérieure & +++ & +++ & - & - & +++ & - & - \\
\hline $\begin{array}{l}\text { Amplitude du mouvement } \\
\text { du condyle }\end{array}$ & ++ & +++ & - & - & + & ++ & ++ \\
\hline Adhérences & - & +++ & & - & +++ & - & - \\
\hline Perfections & - & +++ & - & - & +++ & - & - \\
\hline Tissus mous & +++ & & + & - & +++ & - & - \\
\hline Configuration discales & +++ & +++ & ++ & - & +++ & - & - \\
\hline Maladies dégénératives & ++ & ++ & +++ & + & +++ & ++ & ++ \\
\hline
\end{tabular}

latérale. Le syndrome de Treacher Collins ne doit pas être confondu avec une microsomie : les microsomies présentent constamment un degré variable d'asymétrie alors que le syndrome de Treacher Collins est une anomalie symétrique qui se traduit au niveau mandibulaire par une hypoplasie de la branche [130].

\section{PATHOLOGIE TUMORALE DE L'ATM}

La pathologie tumorale de l'articulation temporo-mandibulaire offre une diversité qui rend impossible l'approche exhaustive dans ce travail. Elle rassemble en effet, les tumeurs proprement articulaires (chondromes synoviaux, ostéochondromatoses synoviales, chondroblastomes bénins etc.) les tumeurs bénignes odontogènes ou non odontogènes des maxillaires localisée à l'ATM, les tumeurs malignes primitives de I'ATM et les tumeurs métastatiques de l'ATM. Enfin, les tumeurs bénignes ou malignes de voisinage qui, de la fosse cérébrale moyenne, de la fosse infra temporale ou de la région parotidienne, peuvent venir interférer avec la physiologie articulaire.

L'IRM joue un rôle majeur dans le diagnostic et plus encore dans le bilan d'extension de ces tumeurs qui envahissent souvent les parties molles peu visualisables en TDM.

\section{CONCLUSION}

La diversité pathologique concernant I'ATM, entité anatomique enfouie, oblige le praticien à bien cibler son mode d'exploration. Les possibilités que nous offrent la technologie moderne permettent de tirer profit et de poser le diagnostic le plus circonstancié dans l'intérêt du patient. Dans cette optique, nous avons rassemblé sous forme de tableau (1) les différents modes d'exploration de I'ATM et leurs indications pour le diagnostic d'une pathologie précise. L'appréciation de ces différentes méthodes va de +++ (bonne), ++ (moyenne), + (très moyenne) à - (mauvaise). Ces appréciations restent néanmoins autant subjectives que temporaires. 


\section{RÉFÉRENCES}

1- B LоCH F. Nuclear Induction. Phys Rev 1946; 70: 7-8.

2 - Purcell EM. Resonance Absorption by Nuclear Magnetic moments in a solid. Phys Rev 1946; 69: 37-8.

3 - Damadian R. Tumor detection by NMR. Science 1971, 178, 1151-3.

4 - Helms CA, Richardson ML, Moon KL, Ware Wh. Nuclear magnetic resonance imaging of the temporomandibular joint: preliminary observation. J Cranionmandib Pract 1984; 2: 220-4.

5 - Hansson LG, Westesson PL, Katzberg RW, Tallents RH, Kurita K, Holtas S, SXEnsSON SA, ERIKSSSON L, J OHANSEN CC. MR imaging of temporomandibular joint: comparison of images of autopsy specimens made at $0.3 \mathrm{~T}$. and $1.5 \mathrm{~T}$. with anatomic cryosections. Am J Roentgenol 1989; 152: 1241-4.

6 - Harms SE, WiLK RM, Wolford LM, Chiles DG, MILAN SB. The temporomandibular joint: magnetic resonance imaging using surface coils. Radiology 1985; 57: 133-6.

7 - Katzberg RW, Bessette RW, Tallents RH, Plewes DB, Manzione J V, SChenck J , Foster TH, HART HR. Normal and abnormal temporomandibular joint: MR imaging with surface coil. Radiology 1986; 158: 183-9.

8 - Spitzer WJ, König H, Meissner R. Hight-resolution magnetic resonance imaging of the temporomandibular joint with particular emphasis on soft-tissus display. Electromedica 1986; 54: 109.

9 - Middleton WD, Macrander S, Lawson TL. Hight resolution surface coil magnetic resonance imaging of the joints: anatomic correlation. Radio Graphics 1987; 7: 645-58.

10 - Wilk RM, Harms SE, Wolford LM. Magnetic resonance imaging of the temporomandibular joint using a surface coil. J Oral Maxillofac Surg 1986; 44: 935-43.

11 - WiLk RM, Harms SE, Wolford LM. Imagerie de l'articulation temporomandibular obtenue par résonance magnétique, avec une antenne de surface: rev Orthop Dento faciale 1988; 22: 447-58.

12 - Donlon WC, Moon KL. Comparison of magnetic resonance imaging, arthrotomograph and clinical and surgical findings in temporomandibular joint derangements. Oral Surg Oral Med Oral Pathol 1987; 64: 2-5.

13 - Requart H, Sauter R, Rayer J, Weber H. Helmholtzspulen in der kernspinyomographie. Electromedica 1987; 55: 61-7.

14 - Hyde J S, Froncisz W, J esmanowicx A, Kneeland J B. Planar-pair local for hight resolution magnetic imaging, particulary of the tempormandibular joint. Med Phys 1986; 13: 1-7.

15 - Hardy CJ, Katzberg RW, Frey RL, Szumowski J, TotTerman S, Mueller OM. Switched surface coil system for bilateral MR imaging. Radiology 1988; 167: 835-8.

16 - WRIGHT SM, WRIGHT RM. Bilateral MR imaging with switched mutually coupled receiver coils. Radiology 1989; 170: 249-55.
17 - Shellock FG, Pressman BD. Dual surface coil MR imaging of bilateral temporomandibular joints: improvements in the imaging protocol. AJ NR. 1989; 10: 595-8.

18 - Fulmer J M, HARMS SE. The temporomandibular joint. Top Magn Reson Imaging 1989; 1: 75-84.

19 - Burnett KR, Davis CL, R EAD J. Dynamic display of the temporomandibular joint meniscus using «fast scan» MR imaging. AJ R 1987; 149; 959-62.

20 - Conway WF, hayes CW, Campbell RL. Dynamic resonance imaging of the temporomandibular joint using flash sequences. J Oral Maxillofac Surg 1988; 46: 930-7.

21 - Vogl TJ, Eberhard D, Bergman C, Lissner J. Incremental hydraulic jaw opener for MR imaging of the temporomandibular joint. J ournal of the magnetic resonance imaging 1992; 2: 479-82.

22 - Avrahami E, Schreiber R, Benmair J, Machtey J, HOROWITZ I. Magnetic resonance imaging of the temporomandibular joint and meniscus dislocation. B ritish J ournal of Radiology 1986; 59: 1153-8.

23 - CARr AB, Gibilisco J A, Berquist TH. Magnetic resonance imaging of the temporomandibular joint: priliminary work. J Craniomand Disord 1987; 1: 89-96.

24 - Dorsay TA, Younberg RA. Cine MRI of the TMJ : need for initial closed mouth images without the burnett device. J Comput Assist Tomogr 1995; 19: 163-4.

25 - Drace J E, Enzmann DR. Defining the normal temporomandibular joint: closed-partially open-an open-mouth MR imaging of the asymptomatic subjetcs. Radiology 1990; 177: 67-71.

26 - Drace J E, Young SW, Enzmann DR. TMJ meniscus and bilaminar zone: MR imaging of the substructuredisgnostic landmarks and pitfalls of interpretation. Radiology 1990; 177: 73-6.

27 - KatzBerg et all. Normal and anormal TMJ : MR imaging with surface coil. Radiology 1986; 54: 109.

28 - Sanchez-woodworth RE, Tallents RH, Katzberg RW, GUAY J A. Bilateral internal derangements of temporomandibular joint: evaluation by magnetic resonance imaging. J Oral Surg Oral Med Oral Pathol 1988; 65: 281-5.

29 - Orwig DS, Helms CA, Doyle GW. Optimal mouth position for magnetic resonance imaging of temporomandibular joint disk. J Craniomandib Disord 1989 ; 3: 138-42.

30- Westesson P, Kwok E, Barsotti J B, hatala M, PAESANI D. Temporomandibular joint: improved MR image quality with decreased section thickness. Radiology 1992; 182: 280-2.

31 - Rao VM, Liem MD, Farole A, Razek A. Occlusive «stuck» Disk of the TMJ : diagnosis with MR imaging. Radiology 1993; 189: 823-7.

32 - J AHn J A, SChallhas KP. Magnetic resonance imaging of the temporomandibular joint: preliminary evaluation of partial flip angle three-dimensional volume acquisitions against conventional single and multiecho pulse sequence. 1991; 9: 145-51. médecine buccale chirurgie buccale VOL. $8, \mathrm{~N}^{\circ}$ 2002 page 33 
médecine buccale chirurgie buccale
33 - Westesson Pl, Katzberg RW, Tallents RH, SanchezWOODWORTH RE, SVENSSON SA. CT and MR of the temproromandibular joint: comparison with autopsy specimens. Amer J Roentgenol 1987; 148; 1165-71.

34 - Westesson PL, BRonsteIn SL. Temporomandibular joint: comparison of single and double contrast arthrography. Radiology 1987; 164: 65-70.

35 - Westesson Pl, Katzberg RW, tallents RH, SANCHEZ-WOODWORTH RE, SVENSSON SA, ESPELAND MA. Temporomandibular joint: comparison of MR images with cryosectional anatomy. Radiology 1987; 164: 59-64.

36 - KIRK WS. JR. Sagittal magnetic resonance image characteristics and surgical findings of mandibular condyle surface disease in staged internal deragements. J Oral Maxillofac Surg 1994; 52: 64-8.

37 - Katzberg RW, Westesson PL, tallents RH, Anderson R, Kurita K, Manzione J V, Totterman S. Temporomandibular joint: MR assessment of rotational and sideways disk displacements. Radiology 1988; 169: 741-8.

38 - Hasso AN, Christiansen EL. The temporomandibular joint Radiol Clin North Am 1989; 27: 301-14.

39 - Kersten HC, Golding RP, VaKJ J, Van Der Kwat WA. Magnetic resonance imaging of partial temporomandibular joint disc displacement. J Oral Maxillofac Surg 1989; 47: 25-9.

40 - Schwaighofer BW, Tanaka TT, Klein MV, Sartoris DJ , RESNICK D. MRimaging of the temporomandibular joint: a cadaver study of the value of coronal images. Am J Roentgenol 1990; 154: 1245-9.

41 - Musgrave MT, Westesson PL, Manzione JV, KATZBERG RW. Improved magnetic resonance imaging of the temporomandibular joint by oblique scanning planes. Oral Surg Oral Med Oral Pathol 1991; 71: 525-8.

42 - TASAKI MM, Westesson PL. Head and Neck Radiology: Temporomandibular J oint: Diagnostic Accuracy with Sagittal and Coronal MR Imaging. Radiology 1993; 186: 723-9.

43 - Fulmer J M. Temporomandibular joint. Magnetic resonancecimaging. Mosby year book 1992; 1244 67.

44 - Matsuda S, Yoshimura Y, Lin V. Magnetic resonance imaging assessment of the temporomandibular joint in disk displacement. J Oral Maxillofac Surg 1994; 23; 266-70.

45 - Laurell KA, Toolte R, Gunningham R, Beltran J, SIMON D. Magnetic resonance imaging of the temporomandibular joint. Part I: literature review. J Prosth Dent 1987; 58: 83-9.

46 - Laurell KA, Toolte R, Gunningham R, Beltran J, SIMON D. Magnetic resonance imaging of the temporomandibular joint. Part II: comparison with laminographie autopsy, and histologic findings. J Prosth Dent 1987: 58: 211-8.

47 - Laurell Ka, Toolte R, Gunningham R, Beltran J, SIMON D. Magnetic resonance imaging of the temporomandibular joint. Part III : Use of a cephalostat for clinical imaging. J Prosth Dent 1987; 58: 355-9.
48 - Brook SL, Westesson PL, ERIKSSON L, Hansson LG, BARSOTTI J B. Prevalence of osseous changes in the temporomandibular joint of symtomatic persons without internal derangement Oral Surg Oral Med Oral Pathol 1992; 73: 118-22.

49- Grayson B, Cutting C, Bookstein FL, Kim H, MCCARTHY J G. The three-dimensional cephalogram: theory, technique and clinical application. Am J Orthod Dentofacial Orthop 1988; 94: 327-37.

50 - Semmler W. : Grundlagen der klinischen anwendung der MRT in der Zahn-, Mund-und kieferheilkunde. Teil I: Grundprinzipien der MRT. Teil II: Klinische Anwendung der MRT. Dtsch Z Mund-kieferGesichts-chir 1986; 10: 31-59.

51 - Schellhas KP, Wilkes CH, El deeb, Lagrotteria LB, OMLIE MR. Permanent proplast temporomandibular joint implants: MR imaging of destructive complications. Am J Roentgenol 1988; 151: 731-5.

52- SChellhas KP, Wilkes CH, Fritts HM, OMLIE MR, HeITHOFF KB, J AHN J A. temporomandibular joint: MR imaging of internal derangements and postoperative changes : AJ NR 1987; 8: 1093-101.

53 - Schellhas KP, Wilkes CH, Fritts HM, Omlie MR, HeITHOFF KB, J AHN J A. Temporomandibular joint: MR imaging of internal derangements and postoperative changes. AJ NR 1988; 151: 381-9.

54 - Conway WF, Hayes CW, Campbell RL, LASKIN DM. Temporomandibular joint motion: efficacy of fast lowangle shot MR imaging Radiology 1989; 172: 821-6.

55 - Crabe J P, Brooks SL, LILLIE J H. Gradient-echo MR imaging of the temporomandibular joint: diagnostic pitfallcaused by the superficial temporal artery. AJ R 1995; 164: 451-4.

56 - Dewes W, Krahe TH, Lückerath W, Gieseke J. MRTomographie des kiefergelenkes unter anwendung T1-gewichteter Multislice-G radientenechoSequenzen. Fortschr Röntgenstr 1988; 148 : 541-4.

57 - SChELLHAS KP. Temporomandibular joint injuries. Radiology 1989; 173: 211-6.

58 - SCHELLHAS KP. Magnetic resonance imaging: Internal derangement of TMJ : Radiologic staging with clinical surgical pathologic Correlation. Radiology 1989; 7: 495-515.

59 - SCHeLLhAS KP. MR imaging of muscles of mastication. AJ NR, 1989; 153: 847-55.

60 - SCHELlHAS KP, WILKes CH ; FRITTS HM, OMLIE MR, LAGROTTERIA LB. MR of osteochondritis dissecans and avascular necrosis of the mandibular condyle. Am. J. Roentgenol. 1989; 152: 551-60

61 - SChellhas KP, Wilkes CH, BaKer CC. Facial pain, headache, and temporomandibular joint inflammation. Headache 1989; 29: 229-32.

62 - SChellhas KP, WiLKes CH. Temporomandibular joint inflammation comparison of $\mathrm{Mr}$ fast scaning to $\mathrm{T1}$ and T2 welghted imaging techniques. AJ NR 1989; 10: 589-94. AJ R. 1989 ; 153: 93-8.

63 - Roberts D, SCHenck J, J OSEPh P, Foster T, Hart H, Pettigrew J, Kundel HL, Edelstein W, Haber b. Temporomandibular joint: magnetic resonance imaging. Radiology 1985; 155: 829-30. 
64 - Spitzer WJ, Lenz M, Sauter R. Darstellung des discus articularis der kiefergelenke mit hilfe der kernspintomographie - vorläufige Mitteilung. Dtsch Zanhnarzti. 1986; 41: 693-6.

65 - Vogler J B, Dolan E, M artinez S. Internal derangements of the temporomandibular joint: diagnosis by magnetic resonance imaging. J Craniomand Disord 1987; 1: 157-61.

66 - Katzberg RW, Westesson PL. Magnetic resonance Imaging. Cranio C linics International 1991; 1: 93-116.

67 - Schellhas KP, Wilkes CH, Omlie MR, Block JC, LARSEN JW, IDELKOPE BI. Temporomandibular joint Imaging: Practical application of available technology. Arch Otolaryngol Head Neck Surg 1987; 113: 744-8.

68 - Heffez LB. Imaging of internal derangements and synovial chrondromatosis of the temporomandibular joint. Radiologie Clinics of North America 1993; 31: 149-61.

69 - KöNIG H, SpITZER WJ. Kernspintomographie des Kiefergelenkes. Fortschr Röntgenstr 1986 ; 144: 580.

70 - König H, SAUter R, Schmit R : Kemspintomographische Diagnostik von Gulenkveränderungen. Fortschr. Röntgenstr. 1986; 145: 43-8.

71 - Yulish BS, Lieberman JM, Newman AJ, Bryan PJ, MULOPULOS GP, MODIC MT. J uvenile rheumatoid arthritis: assessment with $M R$ imaging. Radiology 1987; 165: 149-52.

72 - Helms CA, Kaban LB, Mcneill C, Dodson T. Temporomandibular joint: morphology and signal intensity characteristics of the disk at MR imaging. Radiology 1989 ; 172: 817-20.

73 - Smith HJ , Larheim TA, Aspestrand F. Rheumatic and nonrheumatic disease in the temporomandibular joint: gadolinium-enhenced MR imaging. Radiology. 1992; 185: 229-34.

74 - Mikhael MA, Gorey MT, Mikhael M. Enhanced Mr of temporomandibular joint... Is it worthy the cost? The European society for magnetic resonance in medicine and biology. Book of Abstracts ( $p$ 117) 10 th Annual Scientific Meeting and Exhibition. Rome, 1993.

75 - Katzberg RW, Schenck J, Roberts D, Tallents RH, Manzione JV, Hart HR, Foster TH, Wayne WS, BESSETTE RW. Magnetic resonance imaging of the temporomandibular joint meniscus. J Oral Surg Oral Med Oral Pathol 1985; 59: 332-5.

76 - Koolstrajh, Van Eijden, Van Spronsen, Weil S, Valk. Computer assisted estimation of the lines of action of human masticaory muscles reconstructed in vivo by means of magnetic resonance imaging of parallel sections. Archs Oral Biol, 1990; 35: 549-56.

77- Van ElLden, KloK, Weiljs, KoOlstra. Mechanical capabilities of the human jaw muscles srudies with a mathematical model. Archs oral biol. 1988; 33: 81926.

78 - Lam, Hannam, Wood, Fache, Watanabe. Imaging orofacial tissues by magnetic resonance, Oral Surg. Oral Med. Oral Pathol. 1989; 68: 2-8.

79 - Ralph, Leunbach, Kopp. Magnetic resonance imaging of the masseter muscle of the rabbit : Scand J Dent Res 1991; 99: 162-5.
80 - GAN YH., MA XC, WANG J. Magnetic resonance imaging of lateral pterygoid muscle in temporomandibular joint dysfunction syndrome. Chung-Hua-Kou-ChiangHsueh-Tssa-Chih. 1994 Nov: 29: 326-8, 383.

81- MCMillan, Hannam. Location of needle electrode recording sites in the human masseter muscle by magnetic resonance imaging. Journal of Neuroscience Methods, 1989; 30: 85-9.

82 - Seltzer SE, Ay-ming Wang, B Oston, Mass. Modern imaging of the masseter muscle: normal anatomy and pathosis on CT and MRI. Oral Surg Oral Med Oral Pathol 1987; 63: 622-9.

83 - HaRtman F, Cucchi G. les dysfonctions crânio-mandibulaires ( $p$ 95). Springer Ed, Paris 1993.

84 - Katzberg RW, Burgener FA. Kernspintomographie des Kiefergelenks. Fortschr Röntgenstr 1985; 145: 38-43.

85 - Kreipke DL, Conces J rdj., Sondhi A, Lappas JC, AuguSTYN GT. Normal and anbnormal temporomandibular joints as demonstrated by magnetic resonance imaging. Acta radiologica diagnosis 1986; 3 : 331-3.

86 - Puj OL J. Le ménisque de l'ATM en IRM. Radiology 1995 Sep; 76: 611-21.

87 - Helms CA, Gillepsy T, Sim RE, Richardson ML. Magnetic resonance imaging of the internal derangement of the temporomandibular joint Radiol Clin North Am. 1986; 24: 189-92.

88 - Krasnow AZ, Collier BD, Kneeland B, Carrera GF, Ryan DE, Gingass D, Sewall S, Hellman RS, Isitman AT, Froncisz W, Jesmanowiez A, Hyde J S. Comparison of high-resolution MRI and SPECT bone scintigraphy for noninvasive. Imaging of the temporomandibular joint. J Nucl Med 1987; 28: 1268-74.

89 - Kaplan PA, Tu HK, Williams SM, Lydiatt DD. The normal temporomandibular joint: MR and arthrographic correlation. Radiology 1987; 165: 177-8.

90 - Raustia AM, Pyhtnen J, Pernu H. Clinical, magnetic resonance imaging and surgical findings in patients with temporomandibular joint disorder a survey of the 47 patients. Rofo-Fortschr-Geb-RontgenstrNeuen-Bildgeb-verfabr. 1994; May; 160: 406-11.

91 - Gabler MJ. Greene CS, Palacios E, Perry HT. Effect of arthroscopie temporomandibular joint surgery on articular disk position. J Craniomandib Disord 1989. 3: 191-202.

92 - Cohen SG, Macafee KA. The use of magnetic resonance imaging to determine splint position in the management of internal derangements of the temporomandibular joint. J Craniomandib Disord 1994; 12: 167-71.

93 - KIRK WS. Magnetic resonance imaging and tomographic evaluation of occlusal appliance treatment for advanced internal derangement of the temporomandibular joint. J Oral Maxillofac Surg 1991; 49: 9-12.

94 - RaO VM, Farole A, Karasick D. Temporomandibular joint dysfunction: correlation of MR imaging, arthrography, and arthroscopy. Radiology 1990; 174: 663-7. médecine buccale chirurgie buccale VOL. $8, \mathrm{~N}^{\circ}$ 2002 page 35 
médecine buccale chirurgie buccale
95 - RaO VM, Barbaria A, Manoharan A, Mandel $\mathrm{S}$ GotTehrer N, Wank $H, G$ rosse $S$. Altered condylar morphology associated with disc displacement in TMJ dysfunction: observation by MRI. Magn. Reson. Imaging 1990; 8: 231-5.

96 - Olivetti L, Grazioli L, Cerri G, Superti G, Gaudana $R$, Fugazzola $C$. Magnetic resonance imaging in study of the temporomandibular joint. II. Pathologic findings Radiologia Medica : 1991; 81: 795-802.

97 - LARHEIM TA. Comparative imaging of the temporomandibular joint: Departement of oral radiology, Faculty of dentistry, University of Oslo, Norway. Periodontoly and Restorative. Dentistery Current Science in Dentistry 1992, 163-169.

98 - $\mathrm{CHOl} \mathrm{BH.,} \mathrm{YOO} \mathrm{J} \mathrm{H.,} \mathrm{LeE} \mathrm{WY.} \mathrm{Comparison} \mathrm{of} \mathrm{magne-}$ tic resonance imaging before and after nonsurgical treatment of closed lock. Oral Surg Oral Med Oral Pathol 1994 sep; 78: 301-5.

99 - Lieberman J M, Hans MG, Rozeneweig G, Goldberg J S, BELLON EM. MR imaging of the juvenile temporomandibular joint: piminary report. Radiology. 1992; 182: 531-4.

100 - KIRCOS LT, O RTENDAHL DA, AleXANDER MS. Magnetic resonance imaging of TMJ . 1986; 65: 286.

101 - De-mot B, Casselman J, Deboever J. Pseudodynamic magnetic resonance imaging in the diagnosis of the temporomandibular joint dysfunction. J Prosthet Dent 1994; Sep, 72: 309-13.

102 - Casselman JW, De-mot B, Declerco C, Pattyn G, Meeus L, Vandevoorde P, Steyaert L, Devos V. L'IRM dynamique de l'articulation temporo-mandibulaire technique et application. Ann Radiol 1990; 33: 379-89.

103 - DORSAY TA, Y OUNGBeRg RA, O RR FE. Cine MRI diagnosis and postherapeutic evaluation of an adherent TMJ disc: a case report. J Oral Maxillofac Surg 1994 Nov; 52: 1220-2.

104 - Cirbus MT, Smilack MS, Beltran J, Simon DC. Magnetic resonance imaging in confirming internal deragement. J Prosth Dent 1987; 57: 488-94.

105 - MOSES IJ, S ARTORIS D, G LASS R, TANAKa T, P OKER I. The effect of artroscopie surgical lysis and lavage of the superior joint space on disc position and mobility. J Oral Maxillofac Surg 1989; 47: 674-80.

106 - Vogl TJ , Eberhard D, Weigl P, AsSAL J, R andzIo J . The use of the cinetechnic in the MRI diagnosis of the temporomandibular joint: Rofofortsch geb rontgenstr Neuen Bidgeb Verfahr 1992; 156: 232-7.

107 - Fugazzolla C, Caudana R, Pregaz M, Morelli N, OlivetTI L, Grazioli L. Magnetic resonance in the study of the temporomandibular joint. I Radiologia medica 1991; 81:787-94.

108 - PRICE C. A method of quantifying disc movement on magnetic resonance images of the temporomandibular joint. Part I: The method. Dentomaxillofac Radiol 1990; 19: 59-66.

109 - REELS LA. The structure and function of the mandibular joint. Brit Dent J 1954; 16: 125-33.

110 - S PITZER WJ. Magnetic resonance imaging of the temporomandibular joint (TMJ) meniscus. Rev Stomatol Chir Maxillofac 1990; 91: 123-5.

111 - GibBS I, Martinez Cruz S, Power T. Postoperative MRI of the temporomandibular joint. The European society for magnetic resonance in medecine and biology. Book of Abstracts (p 116) 10 th Annual Scientific Meeting and Exhibition. Rome, 1993.

112 - Pressman, Schellock, Schames J, Schames M. MR imaging of temporomandibular joint abnormalities associated with cervical hyperextension/hyperflexion (whiplash) injuries. J MRI 1993: 2: 569-74.

113 - Buthiau D. et coll : IRM de I'ATM. (pp. 55-6). Vigot éditeur, Paris, 1994.

114 - Norman J E. Ankylosis of the temporo-mandibular joint, Australian Dental J ournal 1978; 23: 56-66.

115-Rowe NL. Ankylosis of the temporo-mandibular joint. J ournal of the Royal College of Surgery of Edimbourg 1982; 27: 67-79, 167-73, 209-18.

116 - Norman J E, B RamLey P. A textbook and colour atlas of the TMJ (pp. 153-75). Wolfe Medical Publication Ed, Londres, 1990.

117 - Nokes SR, Spritzer CE, Volger J B, Dolan E, KIM AG, M ARTINEZ $S$. Duke university Medical Center. Perforation of the TMJ meniscus : Accuracy of MRI. SMRI : Seventh Annual Meeting Program. Book of Abstracts (p 36) 1989.

118 - OshraIN HI, SACKLER A. Involvement of the temporomandibular joint in a case of rheumatoid arthritis. Oral Surg. 1955; 8: 1039-43.

119-Ogus H. Rhumatoid arthritis of the TMJ. British J ournal of Oral Surgery 1975; 12: 275-84.

120 - Stimson CV, Leban SG. Recurrent ankylosis of the TMJ in patient with psoriasic arthritis. J Oral Maxillofac Surg 1982; 40: 678-80.

121 - Smith HJ, LARHEIM TA, Apastrand F. Rheumatic and non rheumatic desease in the temporo-mandibular joint: gadolinium enhanced MR imaging. Radiology. 1992; 185: 229-34.

122 - SCHeLlhAS KP, PIPER MA, O MLIE MR. Facial skeleton remodeling due to temporomandibular joint degeneration: an imaging study of 100 patients. AJ NR 1990; 11; 541-51.

123 - SCHELLHAS KP, PIPER MA, OMLIE MR. Facial skeleton remodeling due to temporomandibular joint degeration: An imaging study of 100 patients. AJ R 1990; 155: 373-83.

124 - Tash R, Demerrit J, Sze G, Leslie D. Hemifacial spasm: MR imaging features. AJ NR 1991; 12: 839-42.

125-CIACCHELLA, HIGGINS. MR imaging of pterygoid muscle inflammation. Magnetic resonance imaging. 1989; 8: 565-6.

126 - LASKIM DM. Diagnosis of pathology of the temporomandibular joint. Clinical and Imaging perpectives. Radiologic Clinics of North America 1993; 1: 135-47.

127 - Westesson PL, Tallents RH, Katzberg RW, GUAY J A. Radiographic assessment asymetry of the mandible. AJ NR 1994 ; 15: 991-9.

128 - GORDIN, Orlando, Higgins. MRI of unilateral facial hypertrophy. Magnetic resonance imaging. 1989; 7: 565-6.

129 - CONVERSE : Craniofacial microsomia in Reconstructive Plastic Surgery (pp.2359). Saunder edition, Philadelphie, 1977.

130 - Poswillo $D$. The pathogenesis of the first and second branchial arch syndromes. I R Soc Med 1973: 35; 302-28. 